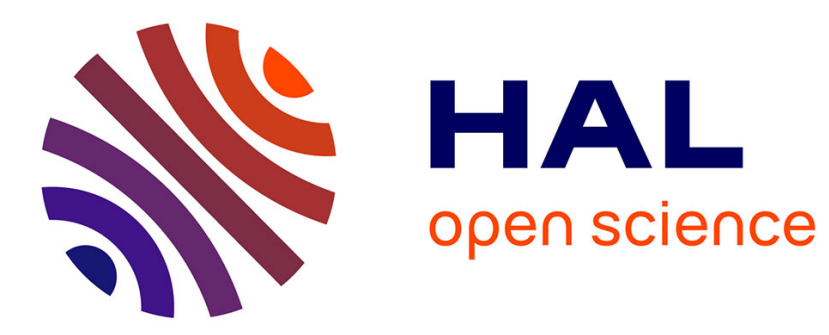

\title{
Remarks on the solution of extended Stokes' problems \\ Giorgio Riccardi
}

\section{To cite this version:}

Giorgio Riccardi. Remarks on the solution of extended Stokes' problems. International Journal of Non-Linear Mechanics, 2011, 10.1016/j.ijnonlinmec.2011.04.010 . hal-00758601

\section{HAL Id: hal-00758601 https://hal.science/hal-00758601}

Submitted on 29 Nov 2012

HAL is a multi-disciplinary open access archive for the deposit and dissemination of scientific research documents, whether they are published or not. The documents may come from teaching and research institutions in France or abroad, or from public or private research centers.
L'archive ouverte pluridisciplinaire HAL, est destinée au dépôt et à la diffusion de documents scientifiques de niveau recherche, publiés ou non, émanant des établissements d'enseignement et de recherche français ou étrangers, des laboratoires publics ou privés. 


\section{Author's Accepted Manuscript}

-

NON-LINEAR

MECHANICS

Remarks on the solution of extended Stokes' problems

Giorgio Riccardi

PII:

S0020-7462(11)00063-1

DOI:

doi:10.1016/j.ijnonlinmec.2011.04.010

Reference:

NLM 1846

To appear in: International Journal of Non-

www.elsevier.com/locate/nlm Linear Mechanics

Received date: 10 August 2010

Revised date: 19 March 2011

Accepted date: 5 April 2011

Cite this article as: Giorgio Riccardi, Remarks on the solution of extended Stokes' problems, International Journal of Non-Linear Mechanics, doi:10.1016/j.ijnonlinmec.2011.04.010

This is a PDF file of an unedited manuscript that has been accepted for publication. As a service to our customers we are providing this early version of the manuscript. The manuscript will undergo copyediting, typesetting, and review of the resulting galley proof before it is published in its final citable form. Please note that during the production process errors may be discovered which could affect the content, and all legal disclaimers that apply to the journal pertain. 


\title{
Remarks on the solution of extended Stokes' problems
}

\author{
Giorgio Riccardi \\ Dept. of Aerospace and Mechanical Engineering, Second University of Naples \\ via Roma, 29 - 81031 Aversa (CE) - Italy \\ email: giorgio.riccardi@unina2.it
}

\begin{abstract}
The analytical solutions of first and second Stokes' problems are discussed, for infinite and finite-depth flows of a Newtonian fluid in planar geometries. Problems arising from the motion of the wall as a whole (one-dimensional flows) as well as of only one half of the wall (two-dimensional) are solved and the wall stresses are evaluated.

The solutions are written in real form. In many cases, they improve the ones in Literature, leading to simpler mathematical forms of velocities and stresses. The numerical computation of the solutions is performed by using recurrence relations and elementary integrals, in order to avoid the evaluation of integrals of rapidly oscillating functions.

The main physical features of the solutions are also discussed. In particular, the steady-state solutions of the second Stokes' problems are analyzed by separating their "in phase" and "in quadrature" components, with respect to the wall motion. By using this approach, stagnation points have been found in infinite-depth flows.
\end{abstract}

keywords: Stokes' flow, first and second Stokes' problems, wall stress, Newtonian fluid, analytical solution.

\section{Introduction}

The analytical solution of Stokes problems for a Newtonian fluid in a planar geometry is here revised, by following the seminal paper of C.M. Liu [1]. A fluid region is bounded by a rigid wall, which moves with a prescribed velocity having fixed direction, parallel to the wall. The fluid and the wall are at rest at the initial time. By following the Literature, wall velocities constant (first Stokes' problem) or periodical (second) in time will be assumed. Moreover, flows in which the wall moves as a whole (one-dimensional) and half wall moves, while the other one is kept fixed, (two-dimensional) will be also investigated. Finally, the depth of the fluid region will be assumed infinite or finite. In these latter kinds of flow, a free surface is assumed to bound the fluid region. 
The solution of the first Stokes' problem in an infinite-depth flow has a well known analytical structure, related to the complementary (real) error function. Solutions of the second problem in an infinite-depth flow have been discussed in [2], [3], [4] and in many other papers. They are usually written in terms of error functions of complex arguments, because in the corresponding real forms integrands containing oscillatory functions appear, the numerical integration of which can lead to severe errors [5].

Recently, these results have been reconsidered in the framework of two-dimensional flows. In the paper [6] the steady states have been found, while Liu [1] generalizes these solutions, by giving also the transient contributions. The effects of side walls on the Stokes flow on a planar wall have been recently investigated in [7]. Besides the first and second Stokes problems, the flows induced by a constant accelerating plate and by a plate that applies a constant stress are also investigated. This important paper opens the way to the comparison with experiments, where effects of side walls are rarely negligible.

Despite the subject is a quite old one [8], many issues about analytical solutions and their numerical computation appear to be improved, in particular for two-dimensional flows. The present paper is an attempt to fill some of these lacks. It is organized as follows. In Section 2, the solutions of one-dimensional first and second problems are briefly discussed, then they are extended to the finite-depth case in Section 3. The solution of two-dimensional problems is then faced, for infinite (Section 4) and finite-depth (Section 5) flows. Finally, conclusions are offered in Section 6.

\section{One dimensional infinite-depth flows}

A Newtonian fluid having kinematical viscosity $\nu$ fills the half space $y>0$, bounded by a solid wall at $y=0$. Initially $(t \leq 0)$, fluid and wall are at rest. The wall starts to move at time $t=0^{+}$with a given velocity (say, $q$ ), directed along the axis $x$. The resulting fluid velocity $(u)$ is assumed to be directed along $x$ and to depend on $y$ and $t$, only. As well known, this flow is described by Stokes' problem:

$$
\left\{\begin{array}{l}
\partial_{t} u=\nu \partial_{y y}^{2} u \\
u(0, t)=q(t), u(+\infty, t) \equiv 0 \\
u(y, 0) \equiv 0
\end{array}\right.
$$




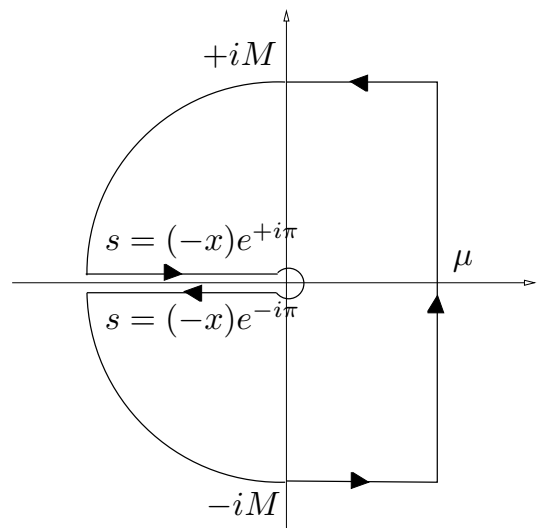

(a)

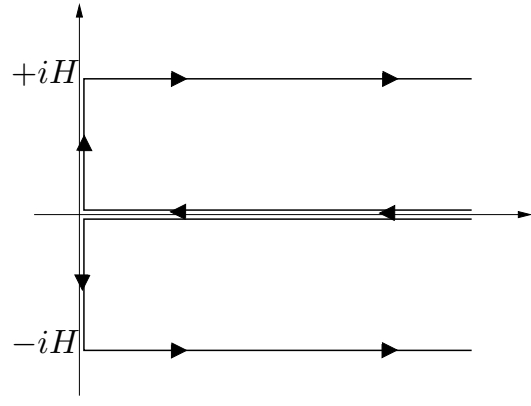

(b)

Figure 1: Integration paths in the plane of $s$ : for the evaluation of the function $F(a)$ and of the integrals $I_{1,2}$.

the solution of which is easily found in terms of Laplace transform in time $\left(q^{(L)}\right.$ and $u^{(L)}$ are the transformed functions of $q$ and $u$, respectively):

$$
u^{(L)}(y, s)=\exp (-\beta y) q^{(L)}(s)
$$

Here, the complex variable $s$ has a positive real part and $\beta=\sqrt{s / \nu}$ (the principal branch of the root is used). In the following, two different wall velocities will be considered: constant, i.e. $q(t) \equiv 0$ as $t<0$ and $q(t) \equiv u_{0}$ as $t>0$, which leads to the first Stokes' problem and periodical, i.e. $q(t)=u_{0} \cos (\omega t+\theta)$ as $t>0$, corresponding to the second Stokes' problem.

\subsection{First Stokes' problem}

The solution of this classical problem is here summarized, for later convenience. The Laplace transform of the wall velocity is:

$$
q^{(L)}(s)=\frac{u_{0}}{s},
$$

so that the time derivative of the nondimensional velocity $U_{1}=u_{1} / u_{0}$ (nondimensional quantities will be indicated by capital symbols, while the subscript 1 refers to the first solution of the present paper) is obtained through a Laplace antitransform of the general solution (1):

$$
\partial_{t} U_{1}=\frac{1}{2 \pi i} \int_{\mu-i \infty}^{\mu+i \infty} d s \exp (t s-y \beta)=: F_{1}
$$

$\mu$ being a suitable positive real number. The function $F_{1}$ is calculated by applying Cauchy's theorem to the integral of $\exp (t s-y \beta) /(2 \pi i)$ on the path of Fig. 1- $a$ and then by performing 
the limit as $M \rightarrow+\infty$. The two resulting integrals are evaluated along the lower and upper paths of Fig. 1-b: it is found that their sum gives $\sqrt{\pi}$. As a consequence, $F_{1}$ assumes the following form:

$$
F_{1}(y, t)=\frac{1}{2 \sqrt{\pi \nu}} y t^{-3 / 2} \exp \left(-\frac{y^{2}}{4 \nu t}\right) .
$$

Once it is inserted in equation (3), an integration in time leads to the classical solution:

$$
U_{1}(Y, T)=\frac{2}{\sqrt{\pi}} \int_{Y /(2 \sqrt{T})}^{+\infty} d \eta e^{-\eta^{2}}=\operatorname{erfc}\left(\frac{Y}{2 \sqrt{T}}\right)
$$

in which lengths and times are nondimensionalized with $\nu / u_{0}$ and $\nu / u_{0}^{2}$, respectively. It can be observed that the velocity (5) depends on $Y$ and $T$ through the time-rescaled variable $Y^{\prime}=Y /(2 \sqrt{T})$ : written in terms of a function of $Y^{\prime}$, the above velocity will be indicated hereafter by $U_{1}^{\prime}\left(Y^{\prime}\right)$. The wall stress $w_{1}$ follows in nondimensional form as $W_{1}=w_{1} /\left(\rho u_{0}^{2}\right), \rho$ being the fluid density. By using the solution (5), one obtains:

$$
W_{1}(T)=-1 / \sqrt{\pi T}
$$

\subsection{Second Stokes problem}

In the second Stokes' problem, the Laplace transform of the wall velocity is:

$$
q^{(L)}(s)=\frac{u_{0}}{2}\left(\frac{e^{-i \theta}}{s+i \omega}+\frac{e^{+i \theta}}{s-i \omega}\right)
$$

so that the general solution (1) is specified in the following one:

$$
\begin{gathered}
U_{2}=\frac{1}{2}\{e^{-i(\omega t+\theta)} \underbrace{\frac{1}{2 \pi i} \int_{\mu-i \infty}^{\mu+i \infty} d s \frac{\exp [t(s+i \omega)-y \beta]}{s+i \omega}}_{H_{2}^{+}}+ \\
+e^{+i(\omega t+\theta)} \underbrace{\frac{1}{2 \pi i} \int_{\mu-i \infty}^{\mu+i \infty} d s \frac{\exp [t(s-i \omega)-y \beta]}{s-i \omega}}_{H_{2}^{-}}\} .
\end{gathered}
$$

The time derivatives of the functions $H_{2}^{ \pm}$are easily evaluated in terms of $F_{1}$, indeed: $\partial_{t} H_{2}^{ \pm}=$ $\exp ( \pm i \omega t) F_{1}$. Once the proper form of the function $F_{1}(4)$ is inserted into the above relations and they are integrated in time, one obtains:

$$
H_{2}^{ \pm}(y, t)=H_{2}^{ \pm}(y, 0)+\frac{y}{2 \sqrt{\pi \nu}} \int_{0}^{t} d \tau \tau^{-3 / 2} \exp \left( \pm i \omega \tau-\frac{y^{2}}{4 \nu \tau}\right)
$$




\section{ACCEPTED MANUSCRIPT}

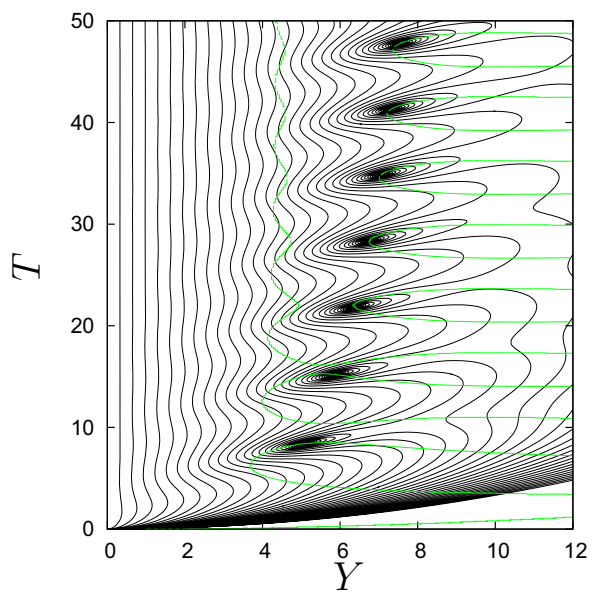

(a)

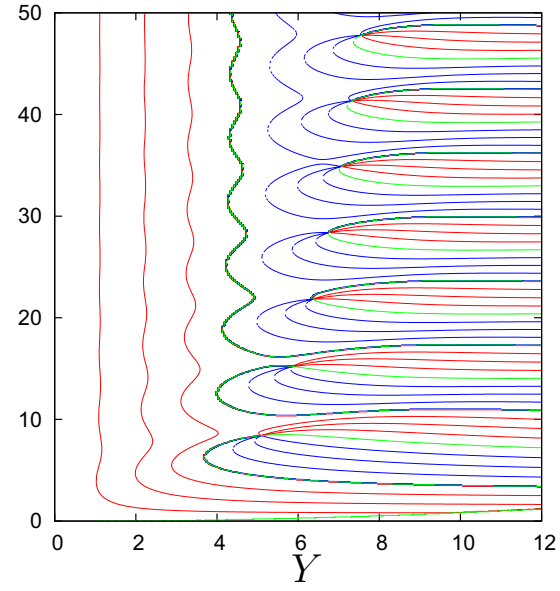

(b)

Figure 2: In $(a)$, the level lines (from -3 to 0 , step 0.1 , black) of $\log _{10} m_{2}$ are superimposed to the line (green) $\varphi_{2}=0^{\circ}$, while in $(b)$ the level lines of $\varphi_{2}$ are drawn (step $45^{\circ}$, red: negative, green: $0^{\circ}$, blue: positive). Notice the presence of stagnation points: in $(a)$ they appear as sinks, while in (b) level lines converge on each of them.

Notice that, in order to have $u_{2}(y, 0) \equiv 0$ for any initial phase $\theta, H_{2}^{ \pm}(y, 0)$ must vanish, as it can be also proved by integrating along the path of Fig. 1- $a$ their definitions (8) evaluated in $t=0$. The functions $H_{2}^{ \pm}(9)$ with $H_{2}^{ \pm}(y, 0) \equiv 0$ are then inserted into the formula (8) and the nondimensional quantities $T=\omega t$ and $Y=y(\omega / \nu)^{1 / 2}$ are used, according to [4]. In this way, the solution:

$$
U_{2}(Y, T)=\frac{2}{\sqrt{\pi}} \int_{Y /(2 \sqrt{T})}^{+\infty} d \eta e^{-\eta^{2}} \cos \left(T+\theta-\frac{Y^{2}}{4 \eta^{2}}\right)
$$

follows. This solution is the real form of the one in [3] for $\theta=0$ and $\theta=\pi / 2$ and of the solution in [4].

The numerical evaluation of the solution (10) is not a trivial task, due to the presence of $1 / \eta^{2}$ in the argument of the trigonometric funtion. Numerical integration schemes leads to a quite poor accuracy, unless huge computational efforts are made. Here, an analytical procedure is proposed, which enables us to easily evaluate the velocity (10). First of all, in a finite precision calculation of the integral:

$$
I_{2}^{c, s}(x):=\frac{2}{\sqrt{\pi}} \int_{x}^{+\infty} d \xi e^{-\xi^{2}} \quad \cos \frac{\alpha}{\sin ^{2}}
$$

( $\alpha$ being a positive constant) the integration range can be extended up to a rather small value $M$ of $\xi$ (e.g., $M \sim 6)$, due to the presence of the factor $\exp \left(-\xi^{2}\right)$ in the integrand function. Then, the interval $(x, M)$ is divided in $N$ sub-intervals $\left(x_{n-1}, x_{n}\right)(n=1,2, \ldots, N)$, with $x_{0}=x$ 
and $x_{N}=M$. By using the above decomposition, $I_{2}^{c, s}$ are rewritten as:

$$
I_{2}^{c, s}=\sum_{n=1}^{N} e^{-x_{n-1}^{2}} \sum_{k=0}^{\infty} J_{2}^{c, s}
$$

in which the integrals:

$$
J_{2}^{c, s}(k):=\frac{(-1)^{k}}{k !} \frac{2}{\sqrt{\pi}} \int_{x_{n-1}}^{x_{n}} d \xi\left(\xi^{2}-x_{n-1}^{2}\right)^{k} \quad \begin{array}{ll}
\cos \frac{\alpha}{\xi^{2}} & \sin
\end{array}
$$

are evaluated through the recurrence formula:

$$
\begin{aligned}
& J_{2}^{c, s}(k)=\frac{1}{2 k+1}\left\{\frac{(-1)^{k}}{k !} \frac{2}{\sqrt{\pi}} x_{n}^{3}\left(x_{n}^{2}-x_{n-1}^{2}\right)^{k-1} \sin \frac{\alpha}{x_{n}^{2}}+\right. \\
& \left.+\frac{1}{k}\left[(4 k-1) x_{n-1}^{2} J_{2}^{c, s}(k-1) \pm 2 \alpha J_{2}^{s, c}(k-1)-2 x_{n-1}^{4} J_{2}^{c, s}(k-2)\right]\right\} .
\end{aligned}
$$

Finally, $J_{2}^{c, s}(0,1)$ are easily computed in terms of Fresnel's integrals $C_{1}$ and $S_{1}$ (see [9] page 300, formulae 7.3.3 and 7.3.4, respectively. About their numerical evaluation, see also Appendix A).

In order to highlight the physical properties of the above solution, the velocity $U_{2}(10)$ is rewritten as sum of a part, say $U_{2}^{c}$, in phase with respect to the wall motion and of another one, $U_{2}^{s}$, which is in quadrature: $U_{2}=U_{2}^{c} \cos (T+\theta)+U_{2}^{s} \sin (T+\theta)$. Moreover, $U_{2}^{c}$ and $U_{2}^{s}$ are assumed as real and imaginary parts of the following complex number: $U_{2}^{c}+i U_{2}^{s}=m_{2} \exp \left(-i \varphi_{2}\right)$ (the branch $-\pi \leq \varphi_{2}<+\pi$ is chosen). Hence, the velocity (10) is also given by the formula:

$$
U_{2}=m_{2} \cos \left(T+\theta+\varphi_{2}\right),
$$

in which $m_{2}$ and $\varphi_{2}$ depend on $Y$ and $T$, but not on $\theta$. The use of the representation (11) enables us to investigate the solution (10) in terms of its amplitude $\left(m_{2}\right)$ and phase relative to the wall motion $\left(\varphi_{2}\right)$, without regard to the initial phase $\theta$.

Level lines of $\log _{10} m_{2}$ and of $\varphi_{2}$ in the plane $(Y, T)$ are drawn in Fig. 2- $a$ and $b$, respectively. At a fixed $T$, the modulus $m_{2}$ increases as $Y$ decreases: it reaches its maximum (1) as $Y \rightarrow 0^{+}$. However, this behaviour appears to be not monotonic, due also to the presence of several points on which $m_{2}$ vanishes: they appear as sinks in the logarithmic scale used in Fig. 2-a. At a fixed $Y, m_{2}$ initially grows with time, afterwards it behaves in an almost periodic way, unless for a discrete set of abscissae $Y$, which correspond to the abovementioned stagnation points. The presence of such points is also confirmed by an inspection to the level lines of the phase $\varphi_{2}$ (Fig. $2-b$ ), that converge on each of them. Fig. 2- $b$ shows that, at $Y$ fixed, two phase behaviours are possible: if the point is quite close to the wall $(Y<4)$, the phase delay reaches an asymptotic 


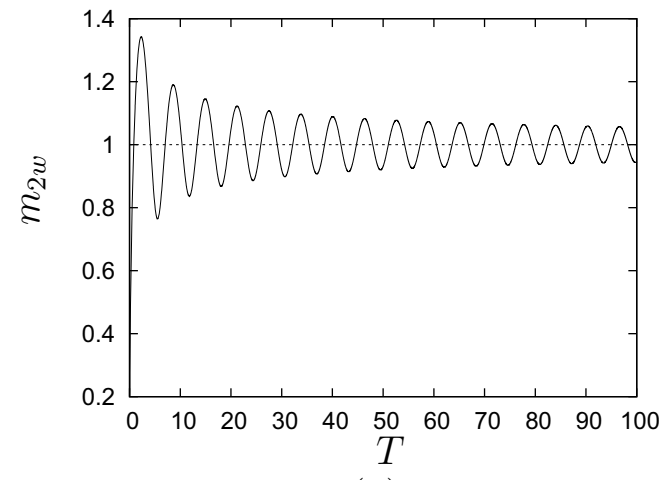

(a)

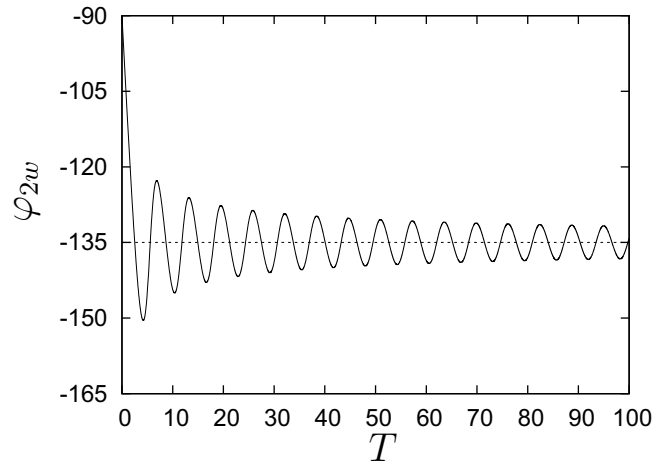

(b)

Figure 3: Modulus $m_{2 w}(a)$ and phase $\varphi_{2 w}$ (degrees) of the wall stress vs. the time $T$ for vanishing initial wall velocity. Asymptotic values are drawn with dashed lines.

value for increasing times, while furthermost points accumulate delays monotonically growing in time.

The wall stress is calculated by noticing that the functions $H_{2}^{ \pm}(9)$ can be rewritten in terms of the complementary error function as:

$$
H_{2}^{ \pm}=e^{ \pm i \omega \tau} \operatorname{erfc}\left(\frac{y}{2 \sqrt{\nu t}}\right) \mp i \omega \int_{0}^{t} d \tau e^{ \pm i \omega \tau} \operatorname{erfc}\left(\frac{y}{2 \sqrt{\nu \tau}}\right)
$$

By deriving in $y$ the above functions and evaluating the resulting derivatives at the wall, the nondimensional wall stress $W_{2}=w_{2} /\left(\rho u_{0} \sqrt{\nu \omega}\right)$ follows:

$$
W_{2}(T)=W_{1}(T) \cos \theta+\sqrt{2}\left[C_{1}(\sqrt{T}) \sin (T+\theta)-S_{1}(\sqrt{T}) \cos (T+\theta)\right] .
$$

The stress (12) is the real form of the one obtained by [4] (see equation (13) of that paper). The first term in the right hand side of equation (12) is due to the initial nonvanishing value $(\cos \theta)$ of the wall velocity and it is the only one which is singular as $T \rightarrow 0^{+}$. The other terms can be rearranged in the form: $m_{2 w} \cos \left(T+\theta+\varphi_{2 w}\right), m_{2 w}$ and $\varphi_{2 w}$ being drawn vs. time in Fig. 3- $a$ and $b$, respectively. Notice the initial overshoot of the modulus $m_{2 w}$ (about $40 \%$ of the asymptotic value 1) and the asymptotic behaviour of the relative phase $\varphi_{2 w}$, which goes to $-3 \pi / 2$ in an oscillatory way as $T \rightarrow \infty$. 


\section{One dimensional finite-depth flows}

As discussed in [1], for a finite-depth flow the Stokes' problem is posed in the following way:

$$
\left\{\begin{array}{l}
\partial_{t} u=\nu \partial_{y y}^{2} u \\
u(0, t)=q(t), \partial_{y} u(h, t) \equiv 0 \\
u(y, 0) \equiv 0
\end{array}\right.
$$

$h$ being the height of the fluid. The second boundary condition enforces vanishing viscous stresses at the interface. Moreover, free surface motion is not considered.

The Laplace transform of the solution is:

$$
u^{(L)}(y, s)=\frac{\cosh [(h-y) \beta]}{\cosh (h \beta)} q^{(L)}(s) .
$$

Notice that the kernel $\cosh [(h-y) \beta] / \cosh (h \beta)$ in equation (13) is an even function of $\beta$, having a countable set of real and negative poles of the first order. They are placed on the points $s_{k}^{(1)}=-K^{2} \nu / h^{2}, K$ being $(k+1 / 2) \pi$ for any non-negative integer $k$.

\subsection{First Stokes' problem}

By inserting the Laplace transform of the wall velocity (2) into the general form of the Laplace transform of the solution (13), the time derivative of the velocity is written as:

$$
\partial_{t} u_{3}=\frac{u_{0}}{2 \pi i} \int_{\mu-i \infty}^{\mu+i \infty} d s e^{s t} \frac{\cosh [(h-y) \beta]}{\cosh (h \beta)}=: u_{0} F_{3}
$$

and the integral in equation (14) is evaluated by applying the residue theorem on the path in Fig. 1-a:

$$
F_{3}=2 \frac{\nu}{h^{2}} \sum_{k=0}^{\infty} K e^{-K^{2} \nu t / h^{2}} \sin (K y / h)
$$

By following [1], the nondimensional variables $T=\nu t / h^{2}$ and $Y=y / h$ are introduced and by accounting for the result:

$$
\sum_{k=0}^{\infty} \frac{\sin (K Y)}{K} \equiv \frac{1}{2}
$$

which holds for $0<Y \leq 1$, the solution of the problem becomes:

$$
U_{3}(Y, T)=1-2 \sum_{k=0}^{\infty} \frac{\sin (K Y)}{K} \exp \left(-K^{2} T\right)=2 \sum_{k=0}^{\infty} K \sin (K Y) \int_{0}^{T} d T^{\prime} e^{-K^{2} T^{\prime}}
$$

The velocity (17) has been obtained in [1]. Here it is also rewritten in an integral form, for later convenience. Notice that the state at $T=0$ is included in the above form of the velocity, 
despite te contrary is stated at page 5 of the abovementioned paper. Indeed, equation (17) gives a velocity $U_{3}(Y, 0)=0$ for any $Y>0$, by using the Fourier series (16). The level lines of the velocity $(17)$ in the plane $(Y, T)$ are drawn in Fig. 4- $a$ : the upper line corresponds to the value $U_{3}=0.95$, while the lower one to $U_{3}=0.05$. It is shown that a large fluid region near the wall (small $Y$ ) accelerates quickly up to velocities near the wall one (1), while the free surface motion is slower: it employes three units of time to reach an almost unitary velocity (see Fig. 4-b).

Finally, the nondimensional wall stress $W_{3}=w /\left(\rho \nu u_{0} / h\right)=\left.\partial_{Y} U_{3}\right|_{Y=0}$ follows from the velocity (17) as:

$$
W_{3}(T)=-2 \sum_{k=0}^{\infty} \exp \left(-K^{2} T\right)
$$

Its behaviour vs. time is shown in Fig. 4-c. It is singular as $T \rightarrow 0^{+}$and becomes negligibly small just after three units of time.

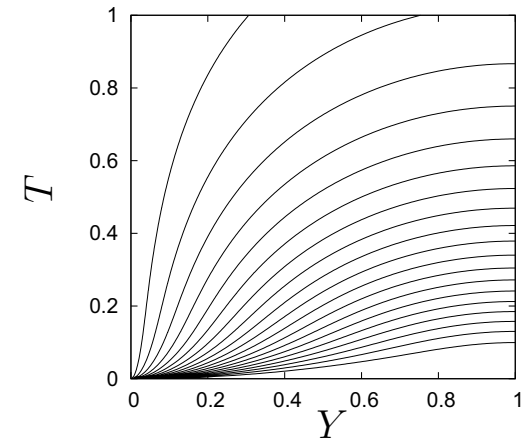

(a)

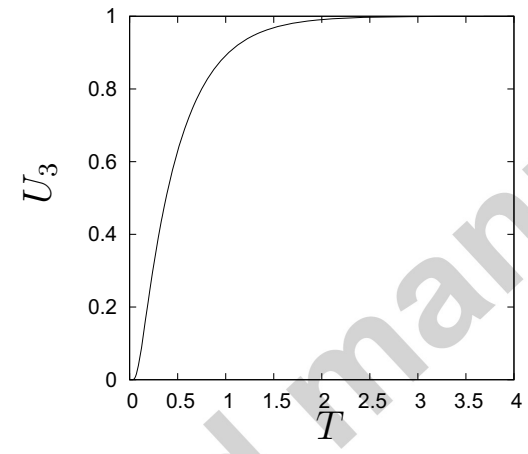

(b)

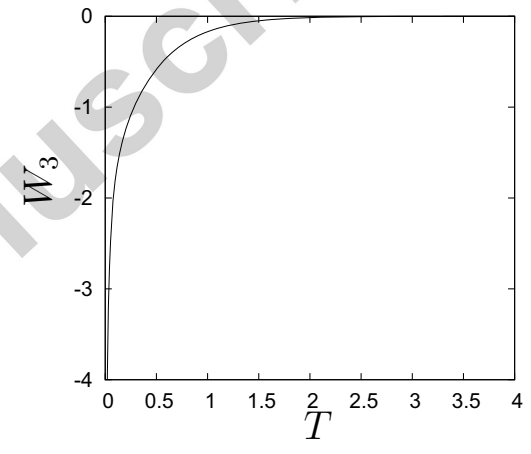

(c)

Figure 4: Level lines of the velocity $(17)$ in the $(Y, T)$ plane ( $a$, from the top to the bottom: $0.95,0.90, \ldots, 0.05)$, free surface velocity $(b)$ and wall stress $(c)$ vs. time.

\subsection{Second Stokes' problem}

Also the second Stokes' problem can be easily extended to finite-depth flows. Indeed, by inserting the Laplace transform of the wall velocity (7) inside the general form of the Laplace 


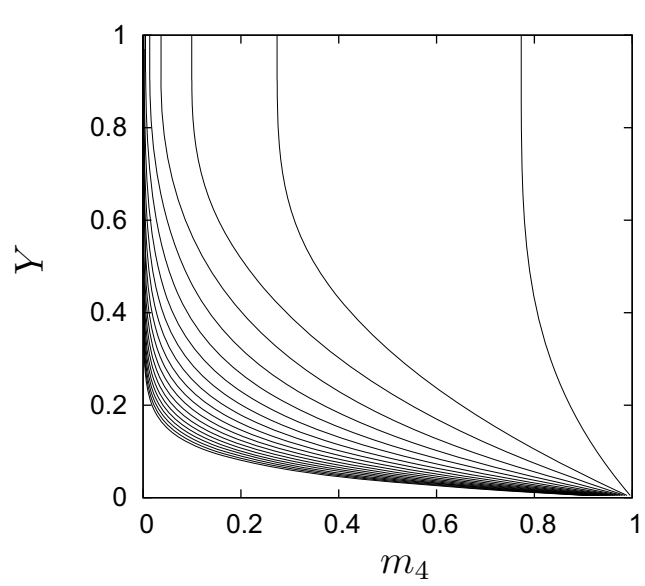

(a)

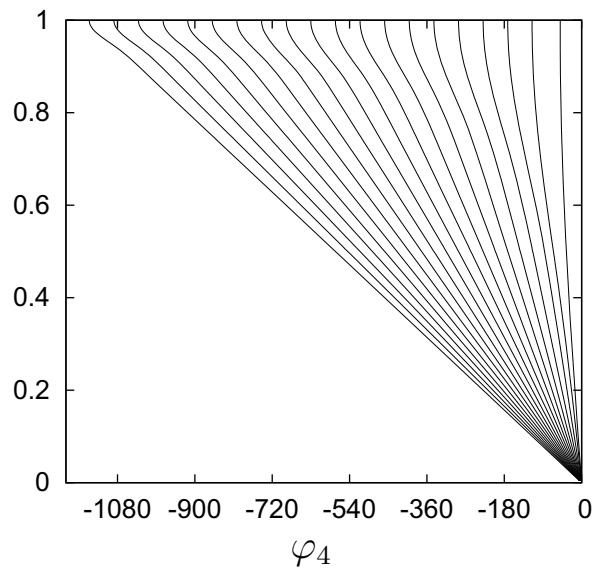

(b)

Figure 5: Profiles of the modulus $(a)$ and of the phase (b, degrees) relative to the wall of the stationary part of the velocity (20). The curves are drawn for different values of $\Omega$, from 1 to 20 with an unitary step. $\Omega$ grows from the right to the left in both figures.

transform of the solution (13), the velocity follows through a Laplace antitransform:

$$
\begin{aligned}
& u_{4}=\frac{u_{0}}{2}\{ e^{-i(\omega t+\theta)} \underbrace{\frac{1}{2 \pi i} \int_{\mu-i \infty}^{\mu+i \infty} d s \frac{e^{(s+i \omega) t}}{s+i \omega} \frac{\cosh [(h-y) \beta]}{\cosh (h \beta)}}_{H_{4}^{-}}+ \\
&+e^{+i(\omega t+\theta)} \underbrace{\frac{1}{2 \pi i} \int_{\mu-i \infty}^{\mu+i \infty} d s \frac{e^{(s-i \omega) t}}{s-i \omega} \frac{\cosh [(h-y) \beta]}{\cosh (h \beta)}}_{H_{4}^{+}}\} .
\end{aligned}
$$

Introduced the nondimensional frequency root $\Omega:=\sqrt{\omega} h / \sqrt{\nu}\left(\Omega^{\prime}:=\Omega / \sqrt{2}\right.$ will be also used), the initial values of the functions $H_{4}^{ \pm}$follow by applying the residue theorem to the integral on the path of Fig. 1- $a$ :

$$
H_{4}^{ \pm}(y, 0)=-2 \sum_{k=0}^{\infty} \frac{K \sin (K y / h)}{K^{2} \mp i \Omega^{2}}+\frac{\cosh \left[\Omega^{\prime}(1-y / h)(1 \mp i)\right]}{\cosh \left[\Omega^{\prime}(1 \mp i)\right]} .
$$

Once the initial values are known, the functions $H_{4}^{ \pm}(y, t)$ are calculated by observing that their time derivatives are related to the function $F_{3}(14)$ through the formula: $\partial_{t} H_{4}^{ \pm}=e^{ \pm i \omega t} F_{3}$, in a complete analogy with the second case of Section 2. The use of the form (15) of $F_{3}$ enables us to calculate $H_{4}^{ \pm}$, for any $t$. As a consequence, in terms of the nondimensional variables $Y=y / h$ and $T=\omega t$, the nondimensional velocity can be written as:

$$
U_{4}(Y, T)=c(Y) \cos (T+\theta)+s(Y) \sin (T+\theta)+
$$




$$
\begin{aligned}
& -2 \sum_{k=0}^{\infty} \frac{K\left(K^{2} \cos \theta+\Omega^{2} \sin \theta\right)}{K^{4}+\Omega^{4}} e^{-K^{2} T / \Omega^{2}} \sin (K Y) \\
= & \frac{2}{\Omega^{2}} \sum_{k=0}^{\infty} K \sin (K Y) \int_{0}^{T} d T^{\prime} e^{-K^{2} T^{\prime} / \Omega^{2}} \cos \left(T+\theta-T^{\prime}\right),
\end{aligned}
$$

in which the two functions $c$ and $s$ are given by the following formulae:

$$
\begin{aligned}
& c(Y)=\frac{\cos \left(\Omega^{\prime} Y\right) \cosh \left[\Omega^{\prime}(2-Y)\right]+\cosh \left(\Omega^{\prime} Y\right) \cos \left[\Omega^{\prime}(2-Y)\right]}{\cosh \left(2 \Omega^{\prime}\right)+\cos \left(2 \Omega^{\prime}\right)} \\
& s(Y)=\frac{\sin \left(\Omega^{\prime} Y\right) \sinh \left[\Omega^{\prime}(2-Y)\right]+\sinh \left(\Omega^{\prime} Y\right) \sin \left[\Omega^{\prime}(2-Y)\right]}{\cosh \left(2 \Omega^{\prime}\right)+\cos \left(2 \Omega^{\prime}\right)} .
\end{aligned}
$$

The form (20) of the solution appears quite satisfactory, the steady-state part being separated by the transient one. The writing of the steady-state part in terms of a Fourier series does not have practical interest, due to its slow convergence velocity, but it enables us to recover the form of the solution given in [1].

As discussed in Section 2, the steady-state velocity is written as the sum of a component in phase with the wall motion and another one which is in quadrature, so that $U_{4}=m_{4} \cos (T+$ $\left.\theta+\varphi_{4}\right)$. The behaviours of the modulus $m_{4}$ and of the phase $\varphi_{4}$ vs. $Y$ are shown in Fig. 5 , for different values of the nondimensional frequency $\Omega$. As expected, the modulus is a decreasing function of the frequency, as the relative phase $\varphi_{4}$ : fluid particles move less and less for increasing frequency. Moreover, they accumulate larger and larger delays.

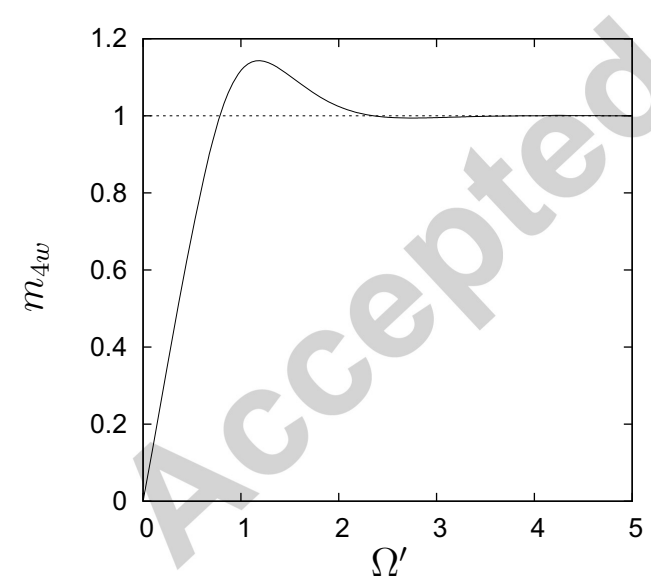

$(a)$

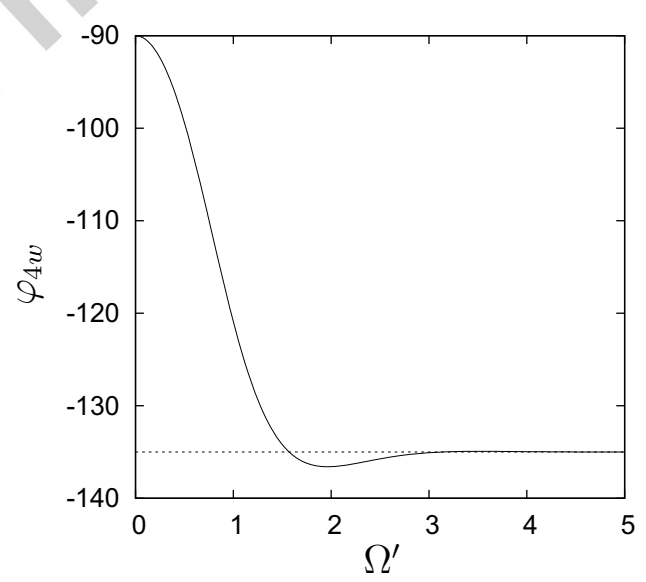

(b)

Figure 6: Modulus $m_{4 w}(a)$ and relative phase $\varphi_{4 w}$ (degrees) of the steady-state wall stress (21) vs. the frequency root $\Omega^{\prime}$. Asymptotic values are also drawn with dashed lines.

The nondimensional wall stress $W_{4}=w_{4} /\left(\rho u_{0} \sqrt{\nu \omega}\right)=\left.\partial_{Y} U_{4}\right|_{Y=0} / \Omega$ follows from the solu- 
tion (20) as:

$$
\begin{aligned}
& W_{4}(T)= \\
= & \frac{W_{3}\left(T / \Omega^{2}\right)}{\Omega} \cos \theta-2 \Omega \sum_{k=0}^{\infty} \frac{K^{2} \sin \theta-\Omega^{2} \cos \theta}{K^{4}+\Omega^{4}} e^{-K^{2} T / \Omega^{2}}+ \\
& -\frac{\sinh \left(2 \Omega^{\prime}\right)-\sin \left(2 \Omega^{\prime}\right)}{\sqrt{2}\left[\cosh \left(2 \Omega^{\prime}\right)+\cos \left(2 \Omega^{\prime}\right)\right]} \cos (T+\theta)+ \\
+ & \frac{\sinh \left(2 \Omega^{\prime}\right)+\sin \left(2 \Omega^{\prime}\right)}{\sqrt{2}\left[\cosh \left(2 \Omega^{\prime}\right)+\cos \left(2 \Omega^{\prime}\right)\right]} \sin (T+\theta) .
\end{aligned}
$$

The first term is due to the nonvanishing initial velocity of the wall (which is just $\cos \theta$ in nondimensional form), while the second one gives the unsteady contribution. The third and fourth terms lead to the steady-state stress, which can be rewritten in the form $m_{4 w} \cos (T+$ $\left.\theta+\varphi_{4 w}\right)$ with:

$$
m_{4 w}=\left[\frac{\cosh \left(2 \Omega^{\prime}\right)-\cos \left(2 \Omega^{\prime}\right)}{\cosh \left(2 \Omega^{\prime}\right)+\cos \left(2 \Omega^{\prime}\right)}\right]^{1 / 2}, \quad \varphi_{4 w}=-\pi+\operatorname{arctg}\left[\frac{\sinh \left(2 \Omega^{\prime}\right)+\sin \left(2 \Omega^{\prime}\right)}{\sinh \left(2 \Omega^{\prime}\right)-\sin \left(2 \Omega^{\prime}\right)}\right] .
$$

The quantities $m_{4 w}$ and $\varphi_{4 w}$ are drawn in Fig. 6 vs. $\Omega^{\prime}$. As expected, the modulus vanishes as $\Omega \rightarrow 0$ and there is a critical frequence such that $m_{4 w}$ reaches its maximum. Moreover, its asymptotic value is unitary. Notice also that the relative phase holds $-\pi / 2$ as $\Omega \rightarrow 0$ and reaches its asymptotic value $(-3 \pi / 4)$ as $\Omega \rightarrow+\infty$.

\section{Two dimensional infinite-depth flows}

A two-dimensional Stokes flow is here considered: half wall $(z>0)$ moves, while half is kept fixed. As a consequence, the fluid velocity $u$ depends on the two spatial variables $y$ and $z$. It is assumed [1] as the sum of the two velocities $u_{o d}(y, t)$ and $u_{t d}(y, z, t)$. They satisfy the following one and two-dimensional problems:

$$
\begin{gathered}
\text { problem for } u_{o d} \\
\left\{\begin{array} { l } 
{ \text { problem for } u _ { t d } } \\
{ \partial _ { t } u _ { o d } = \nu \partial _ { y y } ^ { 2 } u _ { o d } } \\
{ u _ { o d } ( 0 , t ) = q ( t ) / 2 } \\
{ u _ { o d } ( + \infty , t ) \equiv 0 } \\
{ u _ { o d } ( y , 0 ) \equiv 0 }
\end{array} \quad \left\{\begin{array}{l}
\partial_{t} u_{t d}=\nu\left(\partial_{y y}^{2} u_{t d}+\partial_{z z}^{2} u_{t d}\right) \\
u_{t d}(0, z, t)=\operatorname{sign}(z) q(t) / 2 \\
u_{t d}(+\infty, z, t) \equiv 0, \partial_{y} u_{t d}(+\infty, z, t) \equiv 0 \\
u_{t d}(y, \pm \infty, t) \text { finite } \\
u_{t d}(y, z, 0) \equiv 0
\end{array}\right.\right.
\end{gathered}
$$

The problem for $u_{o d}$ has been solved above (with $q$ in place of $q / 2$ ), while the one for $u_{t d}$ will be solved below. 
First of all, the solution $u_{t d}$ is an odd function of $z$, so that the problem can be only posed for $z>0$, by accounting for that $u_{t d}(y, 0, t) \equiv 0$. As before, the Laplace transform in time of the equation of motion leads to the equation: $s u_{t d}^{(L)}=\nu\left(\partial_{y y}^{2} u_{t d}^{(L)}+\partial_{z z}^{2} u_{t d}^{(L)}\right)$. Furthermore, in order to eliminate the derivative in $y$, a Fourier sine transform in $y\left(\mathcal{F}_{s}\right.$, indicated with the apex $\left.{ }^{(F)}\right)$ is also applied, once the following transform:

$$
\mathcal{F}_{s}\left[\partial_{y y}^{2} u_{t d}^{(L)}\right](\eta, z, s)=\eta \frac{q^{(L)}(s)}{2}-\eta^{2} u_{t d}^{(F L)}(\eta, z, s),
$$

has been evaluated. Here, $u_{t d}^{(F L)}$ is the Fourier sine transform of $u_{t d}^{(L)}$. It follows the differential problem:

$$
\left\{\begin{array}{l}
\partial_{z z}^{2} u_{t d}^{(F L)}-\left(\eta^{2}+\beta^{2}\right) u_{t d}^{(F L)}=-\eta q^{(L)} / 2 \\
u_{t d}^{(F L)}(\eta, 0, s) \equiv 0 \\
u_{t d}^{(F L)}(\eta,+\infty, s) \text { finite }
\end{array}\right.
$$

which leads to the general form of the solution:

$$
u_{t d}^{(F L)}(\eta, z, s)=\frac{\eta\left[1-\exp \left(-z \sqrt{\beta^{2}+\eta^{2}}\right)\right]}{\beta^{2}+\eta^{2}} \frac{q^{(L)}(s)}{2} .
$$

The solution (23) will be particularized below for the two aforementioned wall velocities $(2,7)$.

\subsection{First Stokes' problem}

The first Stokes' problem has Laplace transform in time of the wall velocity given by equation (2), so that from the general form of the solution (23) the Fourier sine transform $u_{t d}^{(F)}(\eta, z, t)$ follows:

$$
u_{t d}^{(F)}=\frac{u_{0} \nu \eta}{2} \frac{1}{2 \pi i} \int_{\mu-i \infty}^{\mu+i \infty} d s \frac{1-\exp \left(-z \sqrt{\beta^{2}+\eta^{2}}\right)}{s\left(s+\nu \eta^{2}\right)} e^{s t}=: \frac{u_{0} \nu \eta}{2} F_{5},
$$

$\mu$ being an arbitrary real and positive number. By integrating on the path of Fig. 1- $a$, the time derivative of the new function $F_{5}$ is written as:

$$
\partial_{t} F_{5}=e^{-\nu \eta^{2} t} \operatorname{erf}\left(\frac{z}{2 \sqrt{\nu t}}\right)
$$

to be integrated by starting from the initial value $F_{5}(\eta, z, 0) \equiv 0 . F_{5}$ is then inserted into the form $(24)$ of the velocity $u_{t d}^{(F)}$ and the inverse Fourier sine transform is applied:

$$
u_{t d}=\frac{u_{0}}{4 \sqrt{\pi \nu}} y \int_{0}^{t} d \tau \tau^{-3 / 2} \operatorname{erf}\left(\frac{z}{2 \sqrt{\nu \tau}}\right) \exp \left(-\frac{y^{2}}{4 \nu \tau}\right) .
$$

This velocity is nondimensionalized as in equation (5), by using as length and time scales $\nu / u_{0}$ and $\nu / u_{0}^{2}$, respectively. The solution follows by adding half velocity (5):

$$
U_{5}(Y, Z, T)=\frac{2}{\sqrt{\pi}} \int_{Y /(2 \sqrt{T})}^{+\infty} d \eta e^{-\eta^{2}} \Phi\left(\frac{Z}{Y} \eta\right)
$$


$\Phi(\xi)$ being $[1+\operatorname{erf}(\xi)] / 2$. It corresponds to the solution in [1], unless a different choice of the length scale (here it has been preferred to keep an explicit dependence on time). Notice also that $U_{5}$ (26) goes to $U_{1}$ for $Z \rightarrow+\infty$, while it vanishes for $Z \rightarrow-\infty$. Its numerical evaluation is not a trivial task, it will briefly described below.

In the solution (26), the integral:

$$
I_{5}(x \mid \alpha):=\frac{2}{\sqrt{\pi}} \int_{x}^{+\infty} d \xi \operatorname{erf}(\alpha \xi) e^{-\xi^{2}}
$$

has to be calculated, $\alpha$ and $x$ being real numbers. Notice that $I_{5}(x \mid \pm 1)= \pm\left[1-\operatorname{erf}^{2}(x)\right] / 2$, while if $|\alpha| \neq 1$, the modulus of $\alpha$ can be always assumed smaller than 1 . Indeed, if $|\alpha|>1$ an integration by parts gives the relation: $I_{5}(x \mid \alpha)=\operatorname{sign}(\alpha)-\operatorname{erf}(x) \operatorname{erf}(\alpha x)-I(\alpha x \mid 1 / \alpha)$. Assumed $|\alpha|<1$, the error function in the integral $I_{5}$ is written in a power series and the formula:

$$
I_{5}=\frac{4}{\pi} \sum_{k=0}^{\infty}(-1)^{k} \frac{J_{5}(k)}{2 k+1}
$$

is found, the integrals $J_{5}(k)$ for $k \geq 1$ being evaluated through the recurrence relation:

$$
J_{5}(k):=\frac{\alpha^{2 k+1}}{k !} \int_{x}^{+\infty} d \xi \xi^{2 k+1} e^{-\xi^{2}}=\frac{\alpha}{2} \frac{(\alpha x)^{2 k}}{k !} e^{-x^{2}}+\alpha^{2} J_{5}(k-1),
$$

with $J_{5}(0)=\alpha e^{-x^{2}} / 2$.

As in the case of the velocity $U_{1}(5)$, the solution (26) can be rewritten in a self-similar form: once the time-rescaled variables $Y^{\prime}:=Y /(2 \sqrt{T})$ and $Z^{\prime}:=Z /(2 \sqrt{T})$ have been introduced, the above velocity is given by the new function $U_{5}^{\prime}=U_{5}^{\prime}\left(Y^{\prime}, Z^{\prime}\right)$. In Fig. 7-a the level lines of $U_{5}^{\prime}$ in the plane $\left(Y^{\prime}, Z^{\prime}\right)$ are drawn. As expected, $U_{5}^{\prime}$ vanishes as $Z \rightarrow-\infty$, while it reaches quickly the value $U_{1}^{\prime}\left(Y^{\prime}\right)$, independent of $Z^{\prime}$, as $Z \rightarrow+\infty$. Moreover, a more and more abrupt change from $0(Z<0)$ to $1(Z>0)$ is found as $Y \rightarrow 0$.

In order to calculate the wall stress, the velocity is written in dimensional variables as:

$$
u_{5}=\frac{u_{0} y}{4 \sqrt{\pi \nu}} \int_{0}^{t} d \tau \tau^{-3 / 2}\left[1+\operatorname{erf}\left(\frac{z}{2 \sqrt{\nu \tau}}\right)\right] \exp \left(-\frac{y^{2}}{4 \nu \tau}\right) .
$$

It is derived with respect to $y$ and the change of variable from $\tau$ to $\zeta=1 /(2 \sqrt{\nu \tau})$ into the integrals is performed. If $z<0$, the limit as $y \rightarrow 0^{+}$is directly evaluated by rewriting $1+\operatorname{erf}(z \zeta)$ as $\operatorname{erfc}(|z| \zeta)$. The nondimensional stress $W_{5}=w_{5} /\left(\rho u_{0}^{2}\right)$ :

$$
W_{5}(Z, T)=W_{1}(T)\left[\Phi\left(Z^{\prime}\right)+\frac{\exp \left(-Z^{\prime 2}\right)}{2 \sqrt{\pi} Z^{\prime}}\right]=: W_{1}(T) \Psi\left(Z^{\prime}\right)
$$




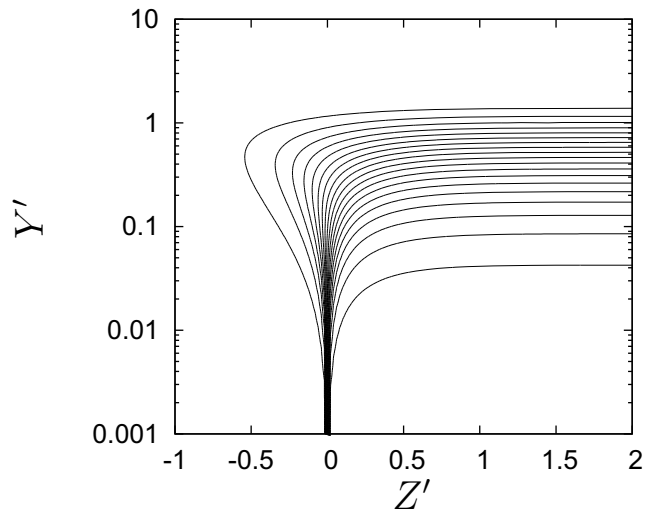

(a)

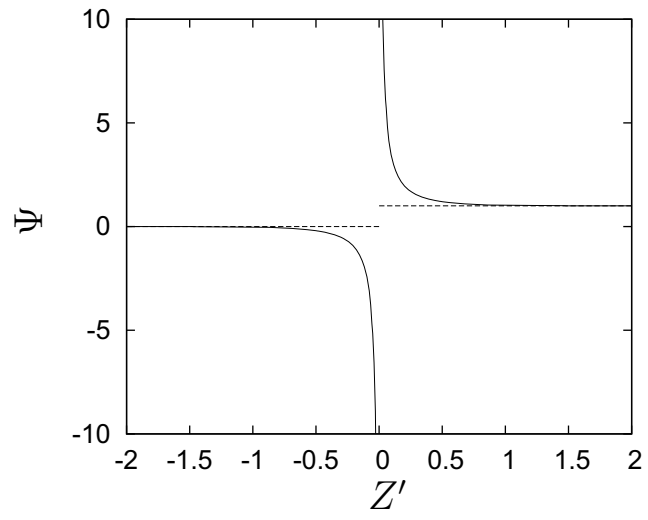

(b)

Figure 7: In (a) the level lines of the velocity (26) (from 0.05 on the upper line to 0.95 on the lower one, with step 0.05) are drawn in the plane of the time-rescaled variables $Y^{\prime}=Y /(2 \sqrt{T})$ (log scale) and $Z^{\prime}=Z /(2 \sqrt{T})$. In $(b), \Psi(27)$ is drawn vs. $Z^{\prime}$ (solid line), together with its asymptotes (dashed): 1 as $Z^{\prime} \rightarrow+\infty$ and 0 as $Z^{\prime} \rightarrow-\infty$.

follows. On the contrary, the limit as $y \rightarrow 0^{+}$cannot be performed on the above form of the derivative when $z$ is positive. In this case the identity: $1+\operatorname{erf}(z \zeta) \equiv-\operatorname{erfc}(z \zeta)+2$ is used and the above form (27) of the stress is recovered. The function $\Psi$ is drawn vs. $Z^{\prime}$ in Fig. 7-b: as expected, the resulting $W_{5}$ is negative for $Z>0$ and positive for $Z<0$. Moreover, it diverges for vanishing $Z^{\prime}$. Notice also that the stress on the moving half plate $(Z>0)$ is everywhere larger than the corresponding stress $W_{1}$ of the one-dimensional case.

\subsection{Second Stokes' problem}

The Fourier sine transform of the solution for the second problem follows by inserting $q^{(L)}(7)$ into the general form of the solution (23) and by Laplace antitransforming:

$$
\begin{array}{r}
u_{t d}^{(F)}=\frac{u_{0} \nu \eta}{4}\{e^{-i(\omega t+\theta)} \underbrace{\frac{1}{2 \pi i} \int_{\mu-i \infty}^{\mu+i \infty} d s \frac{1-\exp \left(-z \sqrt{\beta^{2}+\eta^{2}}\right)}{s+\nu \eta^{2}} \frac{e^{(s+i \omega) t}}{s+i \omega}}_{H_{6}^{+}}+ \\
+e^{+i(\omega t+\theta)} \underbrace{\frac{1}{2 \pi i} \int_{\mu-i \infty}^{\mu+i \infty} d s \frac{1-\exp \left(-z \sqrt{\beta^{2}+\eta^{2}}\right)}{s+\nu \eta^{2}} \frac{e^{(s-i \omega) t}}{s-i \omega}}_{H_{6}^{-}}\} .
\end{array}
$$

It can be shown through integrations on paths like the one in Fig. 1 - $a$ that $H_{6}^{ \pm}(\eta, z, 0)=0$. Hence, equations $\partial_{t} H_{6}^{ \pm}=\exp ( \pm i \omega t) \partial_{t} F_{5}$ and (25) enable us to evaluate the functions $H_{6}^{ \pm}$:

$$
H_{6}^{ \pm}(\eta, z, t)=\int_{0}^{t} d \tau e^{\left(-\nu \eta^{2} \pm i \omega\right) \tau} \operatorname{erf}\left(\frac{z}{2 \sqrt{\nu \tau}}\right)
$$


They are used in $u_{t d}^{(F)}(28)$ and the Fourier sine antitransform is applied:

$$
u_{t d}=\frac{u_{0} \nu}{\pi} \int_{0}^{t} d \tau \operatorname{erf}\left(\frac{z}{2 \sqrt{\nu \tau}}\right) \cos [\omega(t-\tau)+\theta] \int_{0}^{+\infty} d \eta \eta \sin (\eta y) e^{-\nu \tau \eta^{2}}
$$

in which the internal integral holds: $\sqrt{\pi} y \exp \left[-y^{2} /(4 \nu \tau)\right] /\left[4(\nu \tau)^{3 / 2}\right]$. Finally, half velocity (10) is added to $u_{t d} / u_{0}$, in order to obtain the nondimensional solution of the present Stokes' problem:

$$
U_{6}(Y, Z, T)=\frac{2}{\sqrt{\pi}} \int_{Y /(2 \sqrt{T})}^{+\infty} d \eta e^{-\eta^{2}} \cos \left(T+\theta-\frac{Y^{2}}{4 \eta^{2}}\right) \Phi\left(\frac{Z}{Y} \eta\right),
$$

in terms of the nondimensional variables $T=\omega t, Y=y \sqrt{\omega / \nu}$ and $Z=z \sqrt{\omega / \nu}$. As expected, the solution (29) goes to $U_{2}$ as $Z \rightarrow+\infty$ and vanishes as $Z \rightarrow-\infty$. The important issue of its numerically computing will be now discussed.

In the solution (29), the calculation of the following integrals:

$$
I_{6}^{c, s}(x \mid \alpha, \beta):=\frac{2}{\sqrt{\pi}} \int_{x}^{+\infty} d \xi e^{-\xi^{2}} \operatorname{erf}(\beta \xi) \stackrel{\cos }{\sin } \frac{\alpha}{\xi^{2}}
$$

with $\alpha>0$ and any real $\beta$ is needed. As before, due to the presence of the factor $\exp \left(-\xi^{2}\right)$ the integration range can be assumed finite, i.e. from $x$ to $M(M=6$ is used in the present calculations). The integration range is then decomposed in $N$ intervals $\left(x_{n-1}, x_{n}\right)(n=1,2$, $\ldots, N)$ with $x_{0}=x$ and $x_{N}=M$. Hence, the following approximation is considered:

$$
I_{6}^{c, s} \simeq \sum_{n=1}^{N}\left[e^{-x_{n-1}^{2}} \operatorname{erf}\left(\beta x_{n-1}\right) J_{6}^{c, s}(0)+\sum_{k=1}^{\infty} q_{k}\left(x_{n-1}\right) J_{6}^{c, s}(k)\right],
$$

$q_{k}(x)$ being the $k$-th derivative of the function $\exp \left(-\xi^{2}\right) \operatorname{erf}(\beta \xi)$ divided by $k$ ! and calculated in $\xi=x$. Moreover, the integrals $J_{6}^{c, s}(k)(k \geq 3)$ are evaluated through the following recurrence relation:

$$
\begin{aligned}
J_{6}^{c, s}(k):= & \frac{2}{\sqrt{\pi}} \int_{x_{n-1}}^{x_{n}} d \xi\left(\xi-x_{n-1}\right)^{k} \sin \frac{\alpha}{\xi^{2}} \\
= & \frac{2}{\sqrt{\pi}} x_{n}^{3} \frac{\left(x_{n}-x_{n-1}\right)^{k-2}}{k+1} \operatorname{sos} \frac{\alpha}{x_{n}^{2}}-\frac{3 k}{k+1} x_{n-1} J_{6}^{c, s}(k-1)+ \\
& -\frac{3(k-1)}{k+1} x_{n-1}^{2} J_{6}^{c, s}(k-2)-\frac{k-2}{k+1} x_{n-1}^{3} J_{6}^{c, s}(k-3)+ \\
+ & \frac{2 \alpha}{k+1} J_{6}^{-s, c}(k-2)
\end{aligned}
$$

while the first three integrals, say $J_{6}^{c, s}(0,1,2)$, are evaluated in terms of sine and cosine integral functions. 


\section{ACCEPTED MANUSCRIPT}
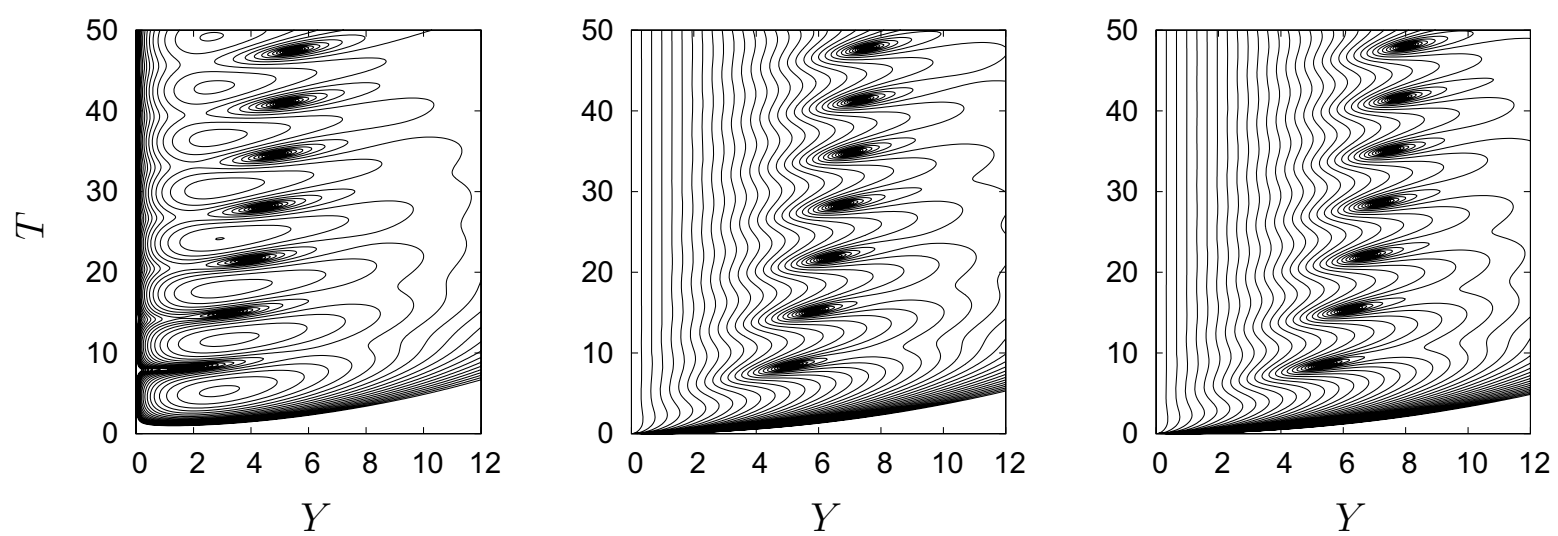

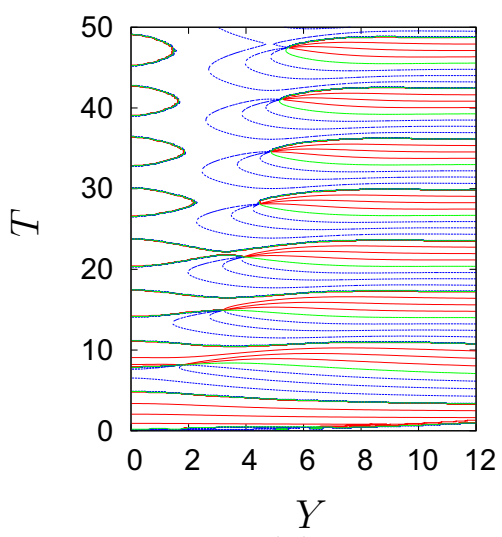

$(a)$

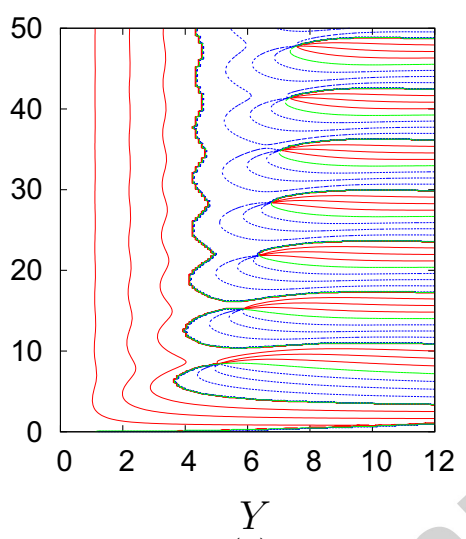

(b)

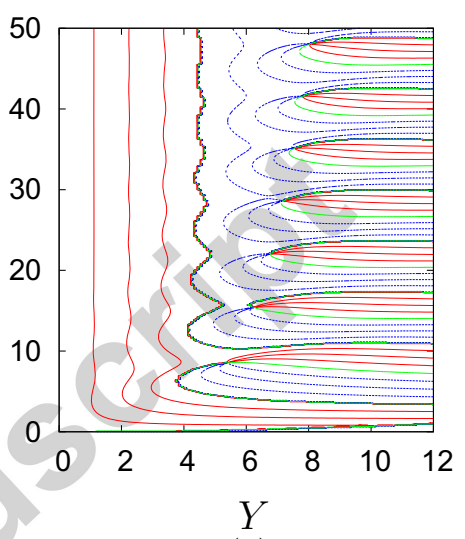

$(c)$

Figure 8: Level lines of the decimal logarithm of the modulus $m_{6}$ (first row) and of the phase $\varphi_{6}$ (second) are drawn in the $(Y, T)$-plane. The coordinate $Z$ holds $-4(a), 0(b)$ and $+4(c)$. The levels of $\log _{10} m_{6}$ are chosen from -4 to 0 with step 0.1 , while the levels of $\varphi_{6}$ are $-180^{\circ},-135^{\circ}, \ldots,+180$. The corresponding lines are drawn with three colours: blue for positive levels, green for the zero one and red for negative $\varphi_{6}$.

Level lines for the modulus $m_{6}$ and the relative phase $\varphi_{6}$ in the $(Y, T)$ plane are shown in Fig. 8 for three values of $Z:-4,0$ and +4 . Relevant differences appear between the fields at $Z=0$ and at $Z=-4$, while the results at $Z=0$ and at $Z=+4$ are quite similar, unless the amplitude of $m_{6}$ which has maximum $1 / 2$ at $Z=0$ and 1 at $Z=+4$, in both cases at the wall $(Y=0)$. On the contrary, the field at $Z=-4$ has maximum about $10^{-2}$ well inside the field, due to the boundary condition of vanishing velocity enforced at the wall. Notice also that the stagnation points are in different positions, nearer to the wall, with respect to the corresponding ones for $Z \geq 0$.

The nondimensional stress $W_{6}=w_{6} /\left(\rho u_{0} \sqrt{\nu \omega}\right)$ reduces to $\left.\partial_{Y} U_{6}\right|_{Y=0}$ and is evaluated 


\section{ACCEPTED MANUSCRIPT}

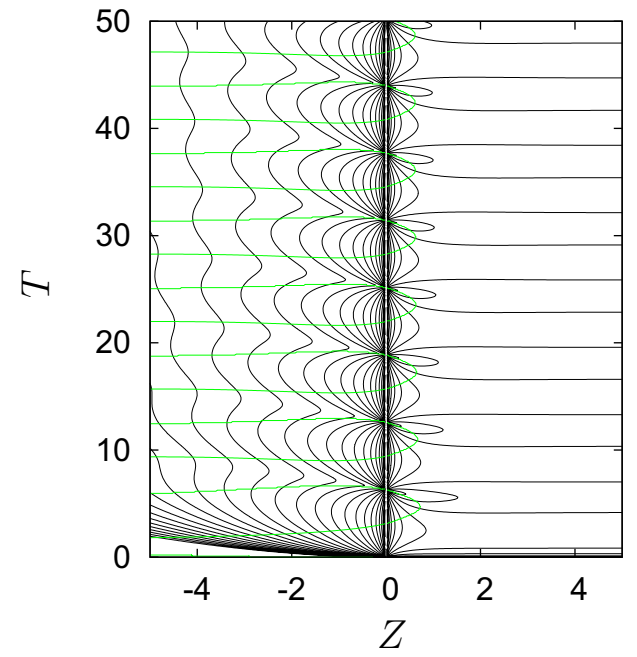

(a)

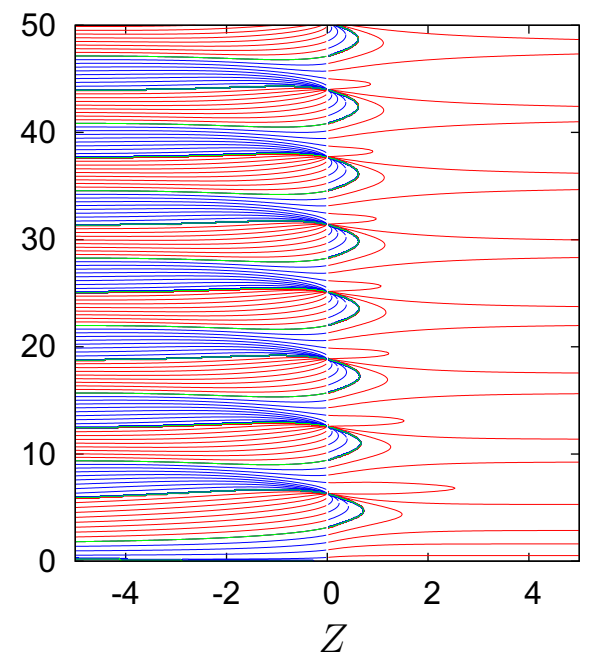

(b)

Figure 9: For vanishing initial wall velocity $(\cos \theta=0)$ in $(a)$ the level lines (from -4 to 1.4, step 0.2 , black) of $\log _{10} m_{6 w}$ are superimposed to the line (green) $\varphi_{6 w}=0^{\circ}$, while in (b) the level lines of $\varphi_{6 w}$ are drawn (step $20^{\circ}$, red: negative, green: $0^{\circ}$, blue: positive).

through repeated integrations by parts as:

$$
\begin{aligned}
W_{6}(Z, T)= & W_{2}(T) \Psi\left(Z^{\prime}\right)+ \\
+ & +\frac{1}{Z} \sqrt{\frac{2}{\pi}}\left[\cos (T+\theta) \int_{0}^{\sqrt{T}} d \xi S_{1}(\xi) e^{-Z^{2} /\left(4 \xi^{2}\right)}-\sin (T+\theta) \int_{0}^{\sqrt{T}} d \xi C_{1}(\xi) e^{-Z^{2} /\left(4 \xi^{2}\right)}\right] .
\end{aligned}
$$

In the numerical computation of the above stress, the main problem lies in evaluating the integrals:

$$
P_{6}^{c, s}(x):=\int_{x_{0}}^{x} d \xi \quad \begin{aligned}
& C_{1} \\
& S_{1}
\end{aligned}(\xi) \exp \left(-\frac{\alpha}{\xi^{2}}\right),
$$

$\alpha$ being a positive constant. The lower bound of integration $x_{0}$ is not vanishing: in the case of the stress (30) it can be set to $|Z| / S$, where $S=12$ in double precision calculations. Then the interval $\left(x_{0}, x\right)$ is divided in $N$ sub-intervals $\left(x_{n-1}, x_{n}\right)(n=1,2, \ldots, N)$ of equal amplitudes and the Fresnel functions are expandend in Taylor series around the point $x_{n-1}$ (see Appendix A). As a consequence, the above integrals are approximated as:

$$
P_{6}^{c, s} \simeq \sum_{n=1}^{N} \sum_{k=0}^{\infty} q_{k}^{c, s}\left(x_{n-1}\right) Q_{6}(k),
$$

in which $q_{k}^{c, s}$ is the $k$-th coefficient of the series for $C_{1}$ or $S_{1}$ and the integral $Q_{6}(k)$ is calculated by the following recurrence formula $(\eta=\sqrt{\alpha} / \xi$ and $k \geq 3)$ :

$$
Q_{6}(k):=\int_{x_{n-1}}^{x_{n}} d \xi\left(\xi-x_{n-1}\right)^{k} \exp \left(-\frac{\alpha}{\xi^{2}}\right)
$$




$$
\begin{aligned}
= & \frac{x_{n}^{3}\left(x_{n}-x_{n-1}\right)^{k-2}}{k+1} e^{-\eta_{n}^{2}}-\frac{3 k}{k+1} x_{n-1} Q_{6}(k-1)+ \\
- & \frac{3(k-1) x_{n-1}^{2}+2 \alpha}{k+1} Q_{6}(k-2)-\frac{k-2}{k+1} x_{n-1}^{3} Q_{6}(k-3) .
\end{aligned}
$$

Finally, the first three integrals $Q_{6}(0,1,2)$ can be easily evaluated in terms of exponential integral and error functions.

In Fig. 9 the level lines of the modulus $m_{6 w}$ and of the relative phase $\varphi_{6 w}$ are drawn in the $(Z, T)$-plane in the case of vanishing initial wall velocity $(\cos \theta=0)$. Notice that $W_{6}$ is not continuous across the line $Z=0$ : the modulus diverges on that line, while the phase change sign. For $Z>0$, the stress reaches quickly the asymptotic value $W_{2}$ (independent of $Z$ ), while it vanishes for $Z \rightarrow-\infty$. In time (at $Z$ fixed) the stress has a nearly periodic behaviour, for $Z$ positive as well as for $Z$ negative, but the phase delay is bounded for $Z>0$, while it grows monotonically for $Z<0$.

\section{Two dimensional finite-depth flows}

A two-dimensional Stokes flow (half plane $y=0$ moves, while half is kept fixed) in a finite-depth fluid is here considered. As before, the solution $u$ is taken as the sum of two velocities: $u_{o d}(y, t)$, which solves a one-dimensional problem, and $u_{t d}(y, z, t)$, that is solution of a two-dimensional one. These problems are stated below:

$$
\begin{gathered}
\text { problem for } u_{o d} \\
\left\{\begin{array} { l } 
{ \partial _ { t } u _ { o d } = \nu \partial _ { y y } ^ { 2 } u _ { o d } } \\
{ u _ { o d } ( 0 , t ) = q ( t ) / 2 } \\
{ \partial _ { y } u _ { o d } ( h , t ) \equiv 0 } \\
{ u _ { o d } ( y , 0 ) \equiv 0 }
\end{array} \quad \left\{\begin{array}{l}
\partial_{t} u_{t d}=\nu\left(\partial_{y y}^{2} u_{t d}+\partial_{z z}^{2} u_{t d}\right) \\
u_{t d}(0, z, t)=\operatorname{sign}(z) q(t) / 2 \\
\partial_{y} u_{t d}(h, z, t) \equiv 0 \\
u_{t d}(y, \pm \infty, t) \text { finite } \\
u_{t d}(y, z, 0) \equiv 0
\end{array}\right.\right.
\end{gathered}
$$

The problem for $u_{o d}$ has been solved above, while the one for $u_{t d}$ will be solved below.

The velocity $u_{t d}$ is an odd function of $z$, so that only the problem with $z>0$ has to be solved, by accounting for the new boundary condition $u_{t d}(y, 0, t) \equiv 0$. Besides the Laplace transform in time, the Fourier sine transform along $z$ will be used: for this reason it will be preferred to work in the difference $u_{o d}-u_{t d}=: v$, rather than in $u_{t d}$. Indeed, $v$ vanishes as $z \rightarrow+\infty$, while the same is not true for $u_{t d}$. To this regard, the Fourier transform of $\partial_{z z}^{2} v$ :

$$
\mathcal{F}_{s}\left[\partial_{z z}^{2} v\right]=\zeta u_{o d}-\zeta^{2} v^{(F)}
$$


will be employed. By accounting for the above result, as well as the problem (31) for $u_{o d}$, it is found that $v^{(F L)}$ satisfies the problem:

$$
\left\{\begin{array}{l}
\partial_{y y}^{2} v^{(F L)}-\left(\beta^{2}+\zeta^{2}\right) v^{(F L)}=-\zeta u_{o d}^{(L)} \\
v^{(F L)}(0, \zeta, s) \equiv 0 \\
\partial_{y} v^{(F L)}(h, \zeta, s) \equiv 0
\end{array}\right.
$$

By introducing the new function $\gamma(s, \zeta):=\left[\beta^{2}(s)+\zeta^{2}\right]^{1 / 2}$, the solution of the above problem is written as:

$$
\begin{aligned}
v^{(F L)}(y, \zeta, s)=\frac{\zeta}{\gamma} & \left\{\frac{\sinh (y \gamma)}{\cosh (h \gamma)} \int_{0}^{h} d \eta \cosh [(h-\eta) \gamma] u_{o d}^{(L)}(\eta, s)+\right. \\
& \left.-\int_{0}^{y} d \eta \sinh [(y-\eta) \gamma] u_{o d}^{(L)}(\eta, s)\right\} .
\end{aligned}
$$

On the other hand, the velocity $u_{o d}^{(L)}$ is given by equation (13) with $q^{(L)} / 2$ in place of $q^{(L)}$, so that the above solution becomes:

$$
v^{(F L)}(y, \zeta, s)=\frac{1}{\zeta}\left\{\frac{\cosh [(h-y) \beta]}{\cosh (h \beta)}-\frac{\cosh [(h-y) \gamma]}{\cosh (h \gamma)}\right\} \frac{q^{(L)}(s)}{2} .
$$

\subsection{First Stokes' problem}

The Laplace transform in time of the wall velocity assumes in this case the form (2). From the general form (32) of the solution it follows:

$$
\frac{v^{(F L)}}{u_{0}}(y, \zeta, s)=\frac{1}{\zeta}\left\{\frac{\cosh [(h-y) \beta]}{\cosh (h \beta)}-\frac{\cosh [(h-y) \gamma]}{\cosh (h \gamma)}\right\} \frac{1}{2 s},
$$

the right hand side of which possesses in the s-plane the following singularities: I ) a branch cut along the interval $\left(-\infty,-\nu \zeta^{2}\right)$, accross which $\gamma$ jumps from (assume $x$ as a point of such an interval) $\gamma=+\gamma^{\prime}=i \sqrt{-x / \nu-\zeta^{2}}$, value that is reached from the above $(s=x+i y$ and $y \rightarrow 0^{+}$), to $\gamma=-\gamma^{\prime}$ from the below; II ) a branch cut along the negative real semi-axis, accross which the function $\beta$ jumps from $\beta=+\beta^{\prime}=i \sqrt{-x / \nu}$ from the above, to $-\beta^{\prime}$ from the below; III) a simple pole in $s=0$; IV ) a countable set of simple poles, which are the zeros of $\left.\cosh (h \beta): s_{k}^{(1)}=-K^{2} \nu / h^{2} ; \mathrm{V}\right)$ a countable set of simple poles, which are the zeros of $\cosh (h \gamma)$ : $s_{k}^{(2)}=-K^{2} \nu / h^{2}-\nu \zeta^{2}$.

The Laplace antitransform in $s$ is performed by integrating on the path in Fig. 1- $a$ (in the limit for $M$ going to infinity), while the evaluation of the residues and of their Fourier sine antitransforms leads to the velocity $u_{t d}$ :

$$
\frac{u_{t d}}{u_{0}}(Y, Z, T)=\sum_{k=0}^{\infty} K \sin (K Y) \int_{0}^{T} d T^{\prime} e^{-K^{2} T^{\prime}} \operatorname{erf}\left(\frac{Z}{2 \sqrt{T^{\prime}}}\right),
$$




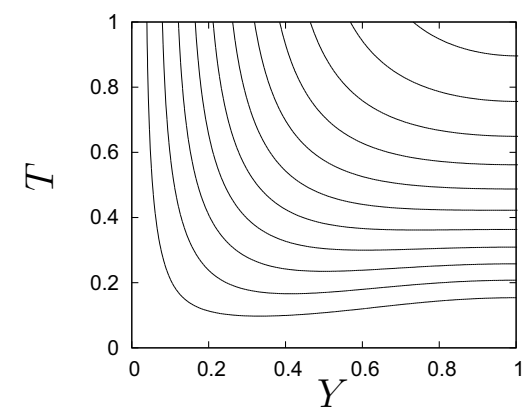

(a)

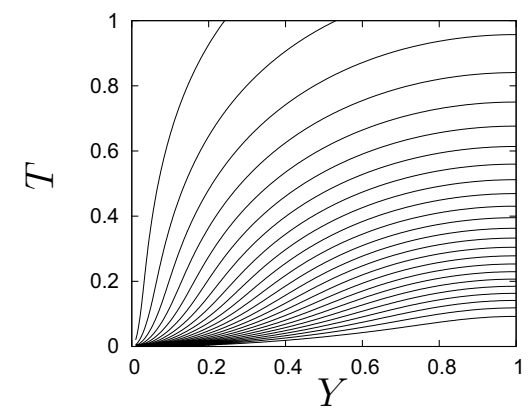

(b)

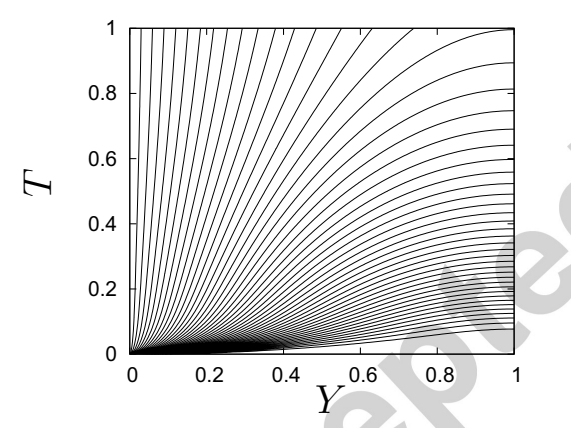

(c)

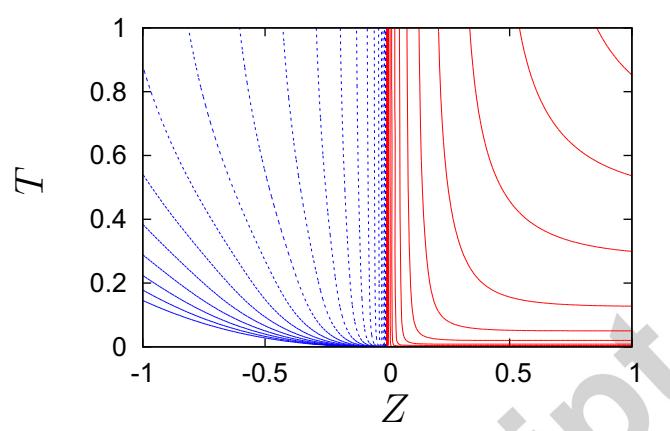

(d)

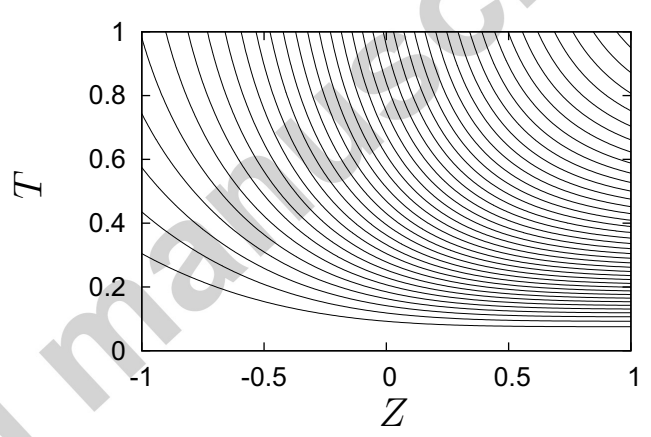

(e)

Figure 10: In $(a, b, c)$ the level lines of $U_{7}$ as a function of $(Y, T)$ at $Z=-0.5,0,+0.5$ are drawn. Levels go from 0.02 to 1 with step 0.02. In $(d)$ the level lines of the wall stress are drawn: positive levels use bleu lines, while red lines are employed for negative levels. Positive levels are in logarithmic scale from 0.01 to $10^{2}$ with logarithmic step 0.2 , while the opposite values are used for negative levels. Finally, in $(e)$ the level lines of the free surface velocity as a function of $Z$ and $T$ are drawn. Levels go from 0.02 to 1 with step 0.02 . 
written in terms of the nondimensional quantities $T=\nu t / h^{2}, Y=y / h$ and $Z=z / h$. Notice that $u_{t d} / u_{0}$ is odd in $Z$ and it goes to $U_{3} / 2$ for $Z \rightarrow+\infty$. The complete nondimensional solution of the problem is obtained as:

$$
\begin{aligned}
U_{7}(Y, Z, T) & =\frac{1}{2}+\sum_{k=0}^{\infty} \frac{\sin (K Y)}{K}\left[K^{2} \int_{0}^{T} d T^{\prime} e^{-K^{2} T^{\prime}} \operatorname{erf}\left(\frac{Z}{2 \sqrt{T^{\prime}}}\right)-e^{-K^{2} T}\right] \\
& =2 \sum_{k=0}^{\infty} K \sin (K Y) \int_{0}^{T} d T^{\prime} e^{-K^{2} T^{\prime}} \Phi\left(\frac{Z}{2 \sqrt{T^{\prime}}}\right) .
\end{aligned}
$$

The above solution possesses the required asymptotic properties in $Z$ : it vanishes as $Z \rightarrow-\infty$, while it goes to $U_{3}$ (17) as $Z \rightarrow+\infty$. Furthermore, the integral can be evaluated by using the formula 7.4 .33 at page 304 of [9]. It follows the solution:

$$
U_{7}(Y, Z, T)=\frac{1+\operatorname{sign}(Z)}{2}+\sum_{k=0}^{\infty} \frac{\sin (K Y)}{K} F_{7}(K \mid Z, T),
$$

where $F_{7}$ is the following function of $K, Z$ and $T$ :

$$
\begin{aligned}
F_{7}(K \mid Z, T)= & -e^{-K^{2} T}\left[1+\operatorname{erf}\left(\frac{Z}{2 \sqrt{T}}\right)\right]-\frac{e^{+K Z}}{2}\left[\operatorname{sign}(Z)-\operatorname{erf}\left(K \sqrt{T}+\frac{Z}{2 \sqrt{T}}\right)\right]+ \\
& -\frac{e^{-K Z}}{2}\left[\operatorname{sign}(Z)+\operatorname{erf}\left(K \sqrt{T}-\frac{Z}{2 \sqrt{T}}\right)\right],
\end{aligned}
$$

$\operatorname{sign}(Z)$ being 0 for $Z=0,+1$ for $Z>0$ and -1 otherwise. Level lines of $U_{7}$ in the plane $(Y, T)$ are drawn in Fig. 10 at different $Z$. In particular, in $(a) Z$ is negative, in $(b)$ vanishes and finally in $(c)$ is positive. In the first case, the velocity vanishes on the axes $(T=0, Y=0)$ and grows for increasing $T$ and $Y$, even if it reaches only quite small values. In correspondence to the plane $Z=0$, the above behaviour changes abruptly, because the fluid in a neighbourhood of the wall (small $Y$ ) moves with velocity about $1 / 2$. For this reason, the level values decrease from the left to the right, as it also occurs at positive $Z(c)$, where larger velocities have been found being $U_{7}(0, Z, T) \equiv 1$. In Fig. 10-e, level lines of the free surface velocity in the $(Z, T)$-plane are also drawn: as expected, $U_{7}$ grows for increasing times, but in a faster way for positive $Z$.

The nondimensional wall stress $W_{7}=w_{7} /\left(\rho \nu u_{0} / h\right)=\left.\partial_{Y} U_{7}\right|_{Y=0}$ follows from the velocity (35) as:

$$
W_{7}(Z, T)=\sum_{k=0}^{\infty} F_{7}(K \mid Z, T)
$$

Level lines of the wall stress (37) in the $(Z, T)$-plane are drawn in Fig. 10- $d$ : it is negative for $Z>0$ and positive for $Z<0$. Moreover, it diverges in correspondence to $Z=0$ and at $T=0$, but only for positive values of $Z$ (for negative $Z$, it vanishes). 


\section{ACCEPTED MANUSCRIPT}

\subsection{Second Stokes' problem}

The use of the Laplace transform $q^{(L)}(7)$ of the wall velocity in the general form of the solution (32) leads to the following solution:

$$
\begin{aligned}
\frac{v^{(F L)}}{u_{0}}(y, \zeta, s)= & \frac{1}{\zeta}\left\{\frac{\cosh [(h-y) \beta]}{\cosh (h \beta)}-\frac{\cosh [(h-y) \gamma]}{\cosh (h \gamma)}\right\} \times \\
& \times \frac{1}{4}\left(\frac{e^{-i \theta}}{s+i \omega}+\frac{e^{+i \theta}}{s-i \omega}\right) .
\end{aligned}
$$

The transformed solution (38) possesses in the $s$-plane the singularities $I, I I, I V$ and $V$ of the first Stokes' problem, together with two simple poles in the points $s= \pm i \omega$.

As before, the Laplace antitransform in $s$ is performed by integrating on the path in Fig. 1-a (in the limit for $M$ going to infinity), while the calculation of the residues and of their Fourier sine antitransforms leads to the following velocity $u_{t d}$ :

$$
\frac{u_{t d}}{u_{0}}(Y, Z, T)=\frac{1}{\Omega^{2}} \sum_{k=0}^{\infty} K \sin (K Y) \int_{0}^{T} d T^{\prime} e^{-K^{2} T^{\prime} / \Omega^{2}} \cos \left(T+\theta-T^{\prime}\right) \operatorname{erf}\left(\frac{\Omega Z}{2 \sqrt{T^{\prime}}}\right)
$$

which is odd in $Z$. Note also that it goes to $U_{4} / 2$ for $Z \rightarrow+\infty$. By adding $U_{4} / 2$ to the nondimensional velocity (39), the following solution is finally obtained:

$$
\begin{aligned}
& U_{8}(Y, Z, T)= \\
= & \frac{1}{2}[c(Y) \cos (T+\theta)+s(Y) \sin (T+\theta)]+ \\
& -\sum_{k=0}^{\infty} \frac{K\left(K^{2} \cos \theta+\Omega^{2} \sin \theta\right)}{K^{4}+\Omega^{4}} \sin (K Y) \exp \left(-K^{2} T / \Omega^{2}\right)+ \\
& +\frac{1}{\Omega^{2}} \sum_{k=0}^{\infty} K \sin (K Y) \int_{0}^{T} d T^{\prime} e^{-K^{2} T^{\prime} / \Omega^{2}} \cos \left(T+\theta-T^{\prime}\right) \operatorname{erf}\left(\frac{\Omega Z}{2 \sqrt{T^{\prime}}}\right) \\
= & \frac{2}{\Omega^{2}} \sum_{k=0}^{\infty} K \sin (K Y) \int_{0}^{T} d T^{\prime} e^{-K^{2} T^{\prime} / \Omega^{2}} \cos \left(T+\theta-T^{\prime}\right) \Phi\left(\frac{\Omega Z}{2 \sqrt{T^{\prime}}}\right) .
\end{aligned}
$$

This solution behaves in the required way for asymptotic values of $Z$. Indeed, it vanished as $Z \rightarrow-\infty$, while it holds $U_{4}$ as $Z \rightarrow+\infty$.

Before discussing the numerical computation of the above solution, it is convenient to integrate by parts inside the second series of the velocity (40) which can be written as follows:

$$
\begin{aligned}
& -\sum_{k=0}^{\infty} \frac{\sin (K Y)}{K} \int_{0}^{T} d e^{-\alpha^{2} T^{\prime}} \cos \left(T+\theta-T^{\prime}\right) \operatorname{erf}\left(\frac{\beta}{\sqrt{T^{\prime}}}\right)= \\
= & \frac{1}{2} \cos (T+\theta) \operatorname{sign}(Z)+\sum_{k=0}^{\infty} \frac{\sin (K Y)}{K} F_{8}(K \mid Z, T),
\end{aligned}
$$




\section{ACCEPTED MANUSCRIPT}

where the new function $F_{8}$ :

$$
\begin{aligned}
& F_{8}(K \mid Z, T)=\int_{0}^{T} d T^{\prime} e^{-\alpha^{2} T^{\prime}} \sin \left(T+\theta-T^{\prime}\right) \operatorname{erf}\left(\frac{\beta}{\sqrt{T^{\prime}}}\right)+ \\
& -\left[I_{8}^{c} \cos (T+\theta)+I_{8}^{s} \sin (T+\theta)\right]-e^{-\alpha^{2} T} \cos \theta \operatorname{erf}\left(\frac{\beta}{\sqrt{T}}\right) .
\end{aligned}
$$

has been introduced. The new quantities used inside the function $F_{8}(42)$ are the two constants $\alpha:=K / \Omega, \beta:=\Omega Z / 2$ and the integrals:

$$
I_{8}^{c, s}(T):=\frac{\beta}{\sqrt{\pi}} \int_{0}^{T} d T^{\prime} e^{-\left(\alpha^{2} T^{\prime}+\beta^{2} / T^{\prime}\right)} T^{\prime-3 / 2} \quad \cos T^{\prime} .
$$

Notice that the integrand function, say $G_{8}^{c, s}\left(T^{\prime}\right)$, vanishes when $\alpha^{2} T^{\prime}$ or $\beta^{2} / T^{\prime}$ are larger than a certain threshold $M^{2}$ (e.g., $\left.M=6\right)$. As a consequence, $G_{8}^{c, s}\left(T^{\prime}\right) \simeq 0$ as $T^{\prime}>T_{\alpha}:=(M / \alpha)^{2}$ (which becomes smaller and smaller as $k \rightarrow \infty$ ) and as $T^{\prime}<T_{\beta}:=(\beta / M)^{2}$. Moreover, one can also replace $\operatorname{erf}\left(\beta / \sqrt{T^{\prime}}\right)$ with $\operatorname{sign}(Z)$ as $T^{\prime}<T_{\beta}$. For this reason, the integral containing $\operatorname{erf}\left(\beta / \sqrt{T^{\prime}}\right)$ becomes an elementary one as $T \leq T_{\beta}$.

Assume $T_{\alpha}>T_{\beta}$, otherwise the above integrals are elementary ones. In this condition, the first integral in the right hand side of equation (41) is splitted in the sum of an integral on $\left(0, T_{\beta}\right)$, which is elementary, and an integral on $\left(T_{\beta}, T\right)$. This latter is reduced to a linear combination of the integrals (43) through an integration by parts:

$$
\begin{aligned}
& \int_{T_{\beta}}^{T} d T^{\prime} e^{-\alpha^{2} T^{\prime}} \sin \left(T+\theta-T^{\prime}\right) \operatorname{erf}\left(\frac{\beta}{\sqrt{T^{\prime}}}\right)= \\
& =\frac{1}{\alpha^{4}+1}\left\{\left(\cos \theta-\alpha^{2} \sin \theta\right) e^{-\alpha^{2} T} \operatorname{erf}\left(\frac{\beta}{\sqrt{T}}\right)+\right. \\
& -\left[\cos \left(T+\theta-T_{\beta}\right)-\alpha^{2} \sin \left(T+\theta-T_{\beta}\right)\right] e^{-\alpha^{2} T_{\beta}} \operatorname{sign}(Z)+ \\
& \left.+\left(I_{8}^{c}+\alpha^{2} I_{8}^{s}\right) \cos (T+\theta)+\left(I_{8}^{s}-\alpha^{2} I_{8}^{c}\right) \sin (T+\theta)\right\} \text {. }
\end{aligned}
$$

The integrals $(43)$ as $T>T_{\beta}(\gamma:=\alpha \beta, x:=|\beta| / \sqrt{T})$ :

$$
I_{8}^{c, s}=\operatorname{sign}(\beta) \frac{2}{\sqrt{\pi}} \int_{x}^{M} d \xi e^{-\xi^{2}} e^{-\gamma^{2} / \xi^{2}} \cos \frac{\beta^{2}}{\xi^{2}}
$$

will be now evaluated. The integration domain is decomposed in a certain number, say $N$, of intervals $\left(x_{n-1}, x_{n}\right)$ and the exponential $\exp \left(-\xi^{2}\right)$ is expanded in Taylor series around the point $\xi=x_{n-1}$. The resulting integrals are evaluated through the recurrence relation $(k \geq 3)$ :

$$
J_{8}^{c, s}(k):=\int_{x_{n-1}}^{x_{n}} d \xi\left(\xi-x_{n-1}\right)^{k} e^{-\gamma^{2} / \xi^{2}} \cos \frac{\beta^{2}}{\sin \frac{\xi^{2}}{2}}
$$




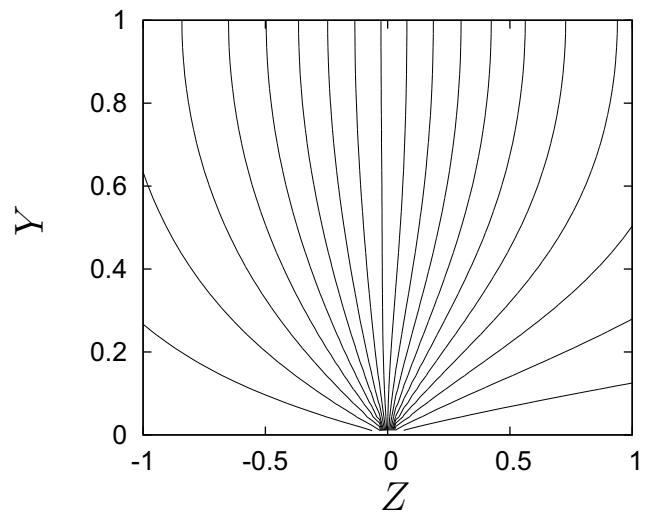

$(a)$

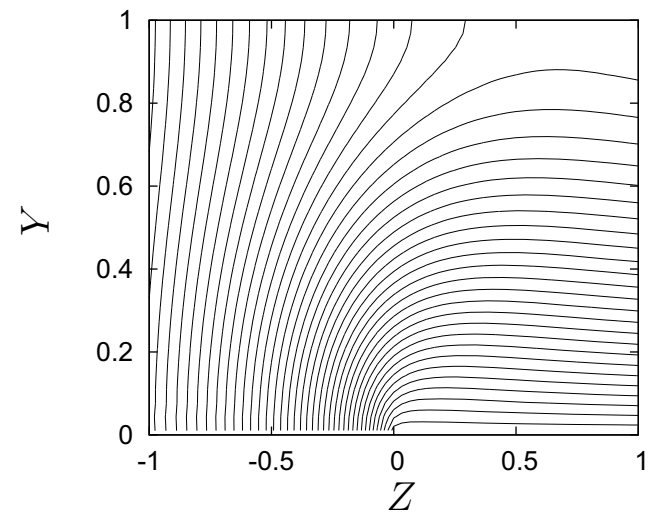

(b)

Figure 11: Level lines of the modulus $m_{8}(a)$ and of the phase $\varphi_{8}(b)$ of the steady-state solution $U_{8}$ in the $(Z, Y)$-plane. $\Omega=1$ is assumed. Steps are 0.05 in $(a)$ and $1^{\circ}$ in $(b)$.

$$
\begin{aligned}
= & \frac{x_{n}^{3}\left(x_{n}-x_{n-1}\right)^{k-2}}{k+1} e^{-\alpha^{2} \eta_{n}} \frac{\cos }{\sin } \eta_{n}+ \\
& -\frac{3 k}{k+1} x_{n-1} J_{8}^{c, s}(k-1)-\frac{3(k-1) x_{n-1}^{2}+2 \gamma^{2}}{k+1} J_{8}^{c, s}(k-2)+ \\
& +\frac{2 \beta^{2}}{k+1} J_{8}^{-s, c}(k-2)-\frac{k-2}{k+1} x_{n-1}^{3} J_{8}^{c, s}(k-3),
\end{aligned}
$$

$\eta$ being $\beta^{2} / \xi^{2}$, while $J_{8}^{c, s}(0,1,2)$ are evaluated in terms of exponential integral or sine and cosine integrals.

Once the problem of numerical computing of the solution (40) has been solved, the behaviour of the steady-state part of the velocity $U_{8}$ is investigated. This part is obtained by setting to 0 terms containing $\exp \left(-K^{2} T / \Omega^{2}\right)$, i.e. the first series in the velocity (40), and by using $\left(0, T_{\alpha}\right)$ as integration range. In Fig. 11 level lines of the modulus $m_{8}(a)$ and of the phase $\varphi_{8}(b)$ are drawn in the $(Y, Z)$-plane. The modulus reaches its maximum 1 on the moving wall $(Y \rightarrow 0$, $Z>0)$ and its minimum 0 on the fixed one $(Y \rightarrow 0, Z<0) . m_{8}(Y ; Z)$ is a monotonically growing function of $Z$ at $Y$ fixed, while it is a decreasing (increasing) function of $Y$ at $Z>0$ $(Z<0)$ fixed. The phase delay with respect to the wall motion $\varphi_{8}$ reaches its maximum 0 at the moving wall and it is a monotonically decreasing function of $Y$, at $Z$ fixed. On the contrary, it is a monotonically growing function of $Z$ at a fixed $Y$, even if its growth becomes smaller and smaller once the free surface is reached.

The nondimensional wall stress $W_{8}=w_{8} /\left(\rho u_{0} \sqrt{\nu \omega}\right)=\left.\partial_{Y} U_{8}\right|_{Y=0} / \Omega$ is evaluated from the 


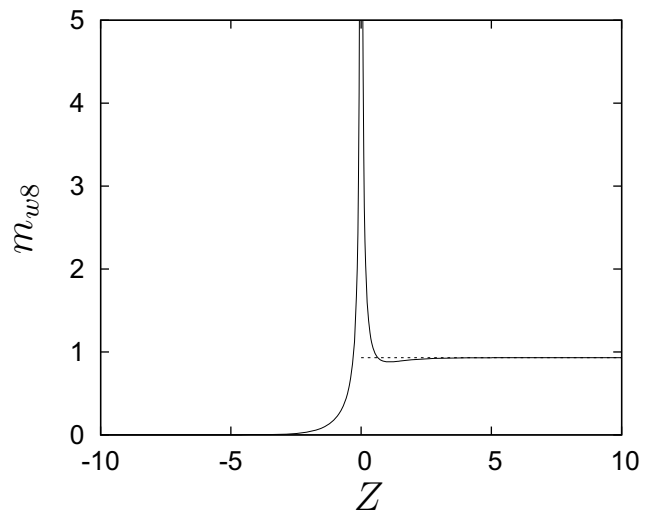

(a)

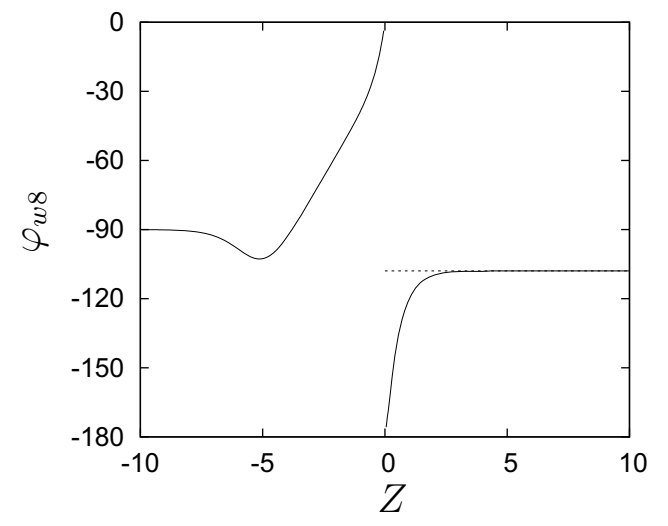

(b)

Figure 12: Modulus $m_{w 8}(a)$ and phase $\varphi_{w 8}(b)$ of the steady-state wall stress $W_{8}$ vs. $Z$. $\Omega=1$ is assumed. Asymptotic values (21) are also drawn with dashed lines.

velocity (40) as:

$$
W_{8}=\frac{W_{4}(T)}{2}+\frac{1}{\Omega} \sum_{k=0}^{\infty} F_{8}(K \mid Z, T) .
$$

In Fig. 12 the modulus $m_{w 8}(a)$ and the phase $\varphi_{w 8}(b)$ of the steady-state stress (45) are drawn vs. $Z$, having assumed $\Omega=1$. As expected, the modulus vanishes as $Z \rightarrow-\infty$, diverges as $Z \rightarrow 0$ and reaches its asymptotic value $(21)$ as $Z \rightarrow+\infty$. Notice also that $m_{w 8}$ is larger than its corresponding one-dimensional value, unless in a rather small interval around $Z=1$. The phase delay $\varphi_{w 8}$ with respect to the wall motion experiences a jump of amplitude $-\pi$ when $Z$ crosses the value 0 . It is not monotonic for negative $Z$, while it monotonically reaches its asymptotic value $(21)$ for positive $Z$. The wall stress is in quadrature with respect to the wall motion as $Z \rightarrow-\infty$, in phase as $Z \rightarrow 0^{-}$and opposite as $Z \rightarrow 0^{+}$.

\section{Conclusions}

The analytical solutions of first and second Stokes' problems in planar geometries have been investigated and the wall stresses are evaluated. Velocity and stress are given in real form. Analytical approaches to the numerical computation of these solutions are also discussed.

In order to investigate the physical properties of the steady-state velocity and wall stress in the case of an oscillating wall (second Stokes' problem), the splitting of the solution in its "in phase" and "in quadrature" components, with respect to the wall motion, is proposed. In this way, amplitude and phase delay of the solution are directly evaluated. Solutions in 
infinite-depth flows exhibit (see Fig. 2 for the one-dimensional problem and Fig. 8 for the two-dimensional one) the presence of stagnation points. It can be argued that a countable set of such points exists, if the entire time interval $(0,+\infty)$ is considered. On the contrary, stagnation points are not found in finite-depth flows.

In many cases, the present solutions have simpler forms than the ones in Literature [1]. Indeed, all the solutions of infinite-depth problems can be posed in the (dimensional) form:

$$
u=u_{0} \frac{y}{2 \sqrt{\pi \nu}} \int_{0}^{t} d \tau \tau^{-3 / 2} \exp \left(-\frac{y^{2}}{4 \nu \tau}\right) P_{1}(t, \tau) P_{2}(z, \tau),
$$

the functions $P_{1,2}$ being defined as:

$$
\begin{aligned}
& P_{1}:= \begin{cases}1 & \text { first problem } \\
\cos [\omega(t-\tau)+\theta] & \text { second problem }\end{cases} \\
& P_{2}:= \begin{cases}1 & 1 D \text { flow } \\
\Phi[z /(2 \sqrt{\nu \tau})] & 2 D \text { flow }\end{cases}
\end{aligned}
$$

Also in finite-depth flows, the same rule appears to be valid. The present solutions have just the form:

$$
u=2 \frac{u_{0} \nu}{h^{2}} \sum_{k=0}^{\infty} K \sin (K y / h) \int_{0}^{t} d \tau e^{-K^{2} \nu \tau / h^{2}} P_{1}(t, \tau) P_{2}(z, \tau) .
$$

In the author opinion, this "unification" of the solutions justifies the use of their real forms. The same is not true for the wall stresses, the analytical forms of which can be hardly related. For example, the presence of integrals of Fresnel's functions $C_{1}$ and $S_{1}$ in $W_{6}$ (30) breaks the symmetry with respect to $W_{5}(27)$, leading to a much more complicated behaviour of the solution.

As stressed by many Authors, the use of the real forms of the solutions leads to severe numerical problems when they are computed in practice, due to the presence of integrals of oscillatory functions. For this reason, complex forms with tabulated functions are often preferred, even if they are much more complicated. In the present paper, integration by series and recurrence formulae are adopted to compute the real forms of the solutions in an accurate and efficient way. With this approach, discretizzation errors are not introduced. The only error sources are due to the propagation of truncation errors along recurrence formulae and to the approximation of series with corresponding finite sums. Both kinds of errors can be handled without difficulties, opening the way to the quantitative use of the real forms of the solutions. 


\section{A Computation of some special functions by series}

Fresnel's integrals can be evaluated by series, once they are treated in order to avoid numerical problems. Indeed, they can be written as:

$$
C_{1}(x)=\sqrt{\frac{2}{\pi}} \int_{0}^{x} d \xi{ }_{\sin }^{\cos } \xi^{2}=\frac{1}{\sqrt{2 \pi}} \int_{0}^{x^{2}} \frac{d \eta}{\sqrt{\eta}} \sin \cos \eta
$$

Assume that $x^{2}$ lies into the interval: $[2 \pi M, 2 \pi(M+1))$ for some non-negative integer $M$. By separating the contributions of each period one obtains:

$$
C_{S_{1}}^{C_{1}}(x)=\frac{1}{\sqrt{2 \pi}}\left[\sum_{m=1}^{M} \int_{0}^{2 \pi} \frac{d \zeta}{\sqrt{\zeta+2 \pi(m-1)}} \sin \zeta+\int_{0}^{x^{2}-2 \pi M} \frac{d \zeta}{\sqrt{\zeta+2 \pi M}} \sin \zeta\right],
$$

where the first sum must be omitted if $M=0$. The above integrals are easily evaluated by series, $\zeta$ being not larger than $2 \pi$. For moderate $m(m<5)$, the series representations of the trigonometric functions are used, so that the above integrals are evaluated through the recurrence ones $(k \geq 1, \alpha=2 \pi(m-1)$ or $2 \pi M)$ :

$$
H_{k}(t ; \alpha)=\frac{1}{k !} \int_{0}^{t} d \xi \frac{\xi^{k}}{\sqrt{\xi+\alpha}}=\frac{1}{k+1 / 2}\left[\frac{t^{k}}{k !} \sqrt{t+\alpha}-\alpha H_{k-1}(t ; \alpha)\right],
$$

with $H_{0}(t ; \alpha)=2(\sqrt{t+\alpha}-\sqrt{\alpha})$. For large $m$, the integrals are rewritten as:

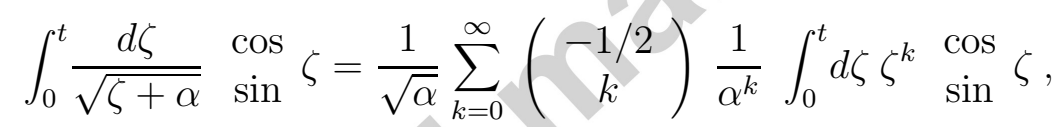

where the trigonometric integrals are easily evaluated. The above procedure has been succesfully tested up to $x=10^{3}$ by finding an excellent agreement with the theoretical asymptotic behaviour [10]. Analogous approaches are used in computing the sine $\operatorname{Si}(x)$ and cosine $\mathrm{Ci}(x)$ integral functions ([9] page 231, formulae 5.2.1 and 5.2.2, respectively).

The exponential integral:

$$
E_{1}(x)=\int_{x}^{+\infty} d \xi \frac{e^{-\xi}}{\xi}
$$

(see [9], page 228 formula 5.1.1) is also computed by expanding in McLaurin series the exponential function in the range $\xi \leq 1$, while in the range $\xi>1$ the following approximation:

$$
E_{1}(x)=\int_{a}^{+\infty} d \xi \frac{e^{-\xi}}{\xi} \simeq \int_{a}^{X} d \xi \frac{e^{-\xi}}{\xi}=\sum_{m=1}^{M} e^{-x_{m-1}} \int_{x_{m-1}}^{x_{m}} d \xi \frac{e^{-\left(\xi-x_{m-1}\right)}}{\xi}
$$

is used ( $a=1$ if $x \leq 1, a=x$ if $x>1$ ). The value $X=33$ is fixed, while a decomposition having constant step $\left(x_{m}-x_{m-1}\right)$ of the order of the unity is used, with $x_{0}=a$ and $x_{M}=X$. The integrals are then evaluated by expanding the exponential functions. 


\section{ACCEPTED MANUSCRIPT}

\section{References}

[1] C.M. Liu. Complete solutions to extended Stokes' problems, Mathematical problems in engineering, 2008: Art. ID 754262, 18 pp., 2008.

[2] R. Panton. The transient for Stokess oscillating plate: a solution in terms of tabulated functions, J. Fluid Mechanics, 31-4: 819 - 825, 1968.

[3] M.E. Erdogan. A note on an unsteady flow of a viscous fluid due to an oscillating plane wall, Int. J. of Non-linear Mech., 35: 1-6, 2000.

[4] C.M. Liu and I.C. Liu. A note on the transient solution of Stokes' second problem with an arbitrary initial phase, J. of Mechanics, 22: $349-354,2006$.

[5] M.E. Erdogan and C.E. Imrak. On the comparison of the solutions obtained by using two different transform methods for the second problem of Stokes for Newtonian fluids, Int. J. of Non-linear Mech., 44: 27 - 30, 2009.

[6] Y. Zeng and S. Weinbaum. Stokes problems for moving half-planes, J. Fluid Mech., 287: $59-74,1995$.

[7] M.E. Erdogan and C.E. Imrak. Some effects of side walls on unsteady flow of a viscous fluid over a plane wall, Math. Problems in Engineering, 2009, Article ID 725196.

[8] H. Schlichting. Boundary layer theory, McGraw-Hill (New York), 1979.

[9] M. Abramowitz and I.A. Stegun. Handbook of Mathematical Functions with Formulas, Graph and Mathematical Tables, Dover (New York), 1965.

[10] E. Kreyszig On the zeros of the Fresnel integrals, Canadian Journal of Mathematics, 9: $118-131,1957$. 


\section{Research Highlights}

- Analytical solutions to first and second Stokes' problems are investigated.

- Solutions are given in real form and their numerical computation is discussed.

- The same analytical structure of the solutions in 1D and 2D flows has been found. 